\title{
Pressure-Enhanced Crystallization Kinetics of Amorphous Si and Ge: Implications for Point- Defect Mechanisms
}

\section{Citation}

Lu, Guo-Quan, Eric Nygren and Michael J. Aziz. 1991. Pressure-enhanced crystallization kinetics of amorphous Si and Ge: Implications for point-defect mechanisms. Journal of Applied Physics 70: 5323-5345.

\section{Published Version}

http://dx.doi.org/10.1063/1.350243

\section{Permanent link}

http://nrs.harvard.edu/urn-3:HUL.InstRepos:3708235

\section{Terms of Use}

This article was downloaded from Harvard University's DASH repository, and is made available under the terms and conditions applicable to Other Posted Material, as set forth at http:// nrs.harvard.edu/urn-3:HUL.InstRepos:dash.current.terms-of-use\#LAA

\section{Share Your Story}

The Harvard community has made this article openly available.

Please share how this access benefits you. Submit a story.

\section{Accessibility}




\section{Pressure-Enhanced Crystallization Kinetics of Amorphous Si and Ge: Implications for Point-Defect Mechanisms}

Guo-Quan Lua), Eric Nygrenb), and Michael J. Aziz, Division of Applied Sciences, Harvard University, Cambridge MA 02138

The effects of hydrostatic pressure on the solid phase epitaxial growth (SPEG) rate, V, of intrinsic Ge (100) and undoped and doped $\mathrm{Si}$ (100) into their respective self-implanted amorphous phases are reported. Samples were annealed in a high-temperature, high-pressure diamond anvil cell. Cryogenically-loaded fluid Ar, used as the pressure transmission medium, ensured a clean and hydrostatic environment. $\mathrm{V}$ was determined by in situ time-resolved visible (for $\mathrm{Si}$ ) or infrared (for Ge) interferometry. $v$ increased exponentially with pressure, characterized by a negative activation volume of $-0.46 \Omega$ in Ge, where $\Omega$ is the atomic volume, and $-0.28 \Omega$ in Si. The activation volume in $\mathrm{Si}$ is independent of both dopant concentration and dopant type. Structural relaxation of the amorphous phases has no significant effect on $\mathrm{V}$. These and other results are inconsistent with all bulk point-defect mechanisms, but consistent with all interface point-defect mechanisms, proposed to date. A kinetic analysis of the Spaepen-Turnbull interfacial dangling bond mechanism is presented, assuming thermal generation of dangling bonds at ledges along the interface, independent migration of the dangling bonds along the ledges to reconstruct the network from the amorphous to the crystalline structure, and unimolecular annihilation kinetics at dangling bond "traps". The model yields

$\mathrm{v}=2 \sin (\theta) \mathrm{v}_{\mathrm{S}} n_{\mathrm{r}} \exp \left(\frac{\Delta S_{\mathrm{f}}+\Delta S_{\mathrm{m}}}{k}\right) \exp -\left(\frac{\Delta H_{\mathrm{f}}+\Delta H_{\mathrm{m}}}{k T}\right)$, where $\Delta S_{\mathrm{f}}$ and $\Delta H_{\mathrm{f}}$ are the standard entropy and enthalpy of formation of a pair of dangling bonds, $\Delta S_{\mathrm{m}}$ and $\Delta H_{\mathrm{m}}$ are the entropy and enthalpy of motion of a dangling bond at the interface, $\mathrm{v}_{\mathrm{S}}$ is the speed of sound, $\theta$ is the misorientation from $\{111\}$, and $n_{\mathrm{r}}$ is the net number of hops made by a dangling bond before it is annihilated. It accounts semi-quantitatively for the measured prefactor, orientation dependence, activation energy and activation volume of $\mathrm{v}$, and the pressure of a "free-energy catastrophe" beyond which the exponential pressure-enhancement of SPEG cannot continue uninterrupted due to a vanishing barrier to dangling bond migration. The enhancement of $v$ by doping can be accounted for by an increased number of charged dangling bonds, with no change in the number of neutrals, at the interface. Quantitative models for the doping dependence of $\mathrm{V}$ are critically reviewed. At low concentrations the data can be accounted for by either the Fractional Ionization or the generalized Fermi-LevelShifting models; methods to further test these models are enumerated. Ion irradiation may affect $\mathrm{V}$ by altering the populations of interfacial dangling bonds or may involve bulk point defects of any type impinging on the interface and converting to dangling bonds, but when the ion beam is turned off, $\mathrm{v}$ cannot be limited by the arrival rate of these suddenly-less-numerous defects. It may also involve alternative point-defect mechanisms operating in parallel with thermal generation of dangling bonds at the interface. 


\section{INTRODUCTION}

One of the outstanding problems in the study of crystal growth is the kinetics of interface motion. Our ability to predict how the growth velocity depends on interface temperature, pressure, composition, orientation, and atomic factors such as molecular structure, interface structure and crystal defect structure is meager. This is in part due to the tremendous complexity of the problem as stated above. One of the conceptually simplest cases to study is the crystallization of an amorphous phase, since the number of orientation variables is greatly reduced and the number of relevant crystal structures is halved. If the system is composed of a single element, it can further simplify the problem by eliminating long range atomic diffusion, interfacial partitioning, compositional short range order and the like from consideration. Of the few elemental amorphous phases, amorphous $\mathrm{Si}(\mathrm{a}-\mathrm{Si})$ and $\mathrm{Ge}(\mathrm{a}-\mathrm{Ge})$ have been studied the most extensively. Crystal growth into a-Si and a-Ge can therefore be considered prototypical of many other crystal growth problems.

Annealing of ion-implantation-amorphized surface layers of $\mathrm{Si}$ and $\mathrm{Ge}^{1-6}$ results in the crystallization of the amorphous phase by solid phase epitaxial growth (SPEG), which occurs by the motion of a sharp crystal/amorphous (c/a) interface toward the free surface. The growth rates are well described by an Arrhenius dependence on temperature, with activation energies of 2.70 and $2.0 \mathrm{eV}$ for $\mathrm{Si}$ and $\mathrm{Ge}$, respectively. The effects of dopants ${ }^{3}$ and ion irradiation ${ }^{7-11}$ on the growth process in Si also have been established. In spite of numerous experiments, many of which have been reviewed recently by Olson and Roth ${ }^{3}$, no agreement yet exists on the atomistic mechanism of the process. Models 2,6,12-17 invoke different types of defects whose creation or transport at or to the c/a interface are proposed as the rate-limiting step in SPEG. In Table I we list several models proposed to explain SPEG in Si.

Variations in pressure, like variations in temperature, are commonly viewed not to affect the nature of the atomistic processes involved, but only their rates. Thus the pressure dependence of a kinetic process, which bears directly on the atomistic mechanism, provides a unique additional parameter for its determination. According to the theory of thermally activated growth ${ }^{18-20}$, the growth rate is generally described by:

$$
\mathrm{v}=A \lambda v \exp \left(-\frac{\Delta G^{*}}{k T}\right)\left[1-\exp \left(\frac{\Delta G^{\mathrm{o}}}{k T}\right)\right]
$$

where $A$ is a geometric factor dependent upon the atomic mechanism, $\lambda$ is the local distance the interface moves per atomic rearrangement, $v$ is an effective normal mode frequency leading to rearrangement ${ }^{21}, \Delta G^{0}$ is the standard Gibbs free energy change per rearrangement (defined to be negative in eq. (1) for crystal growth), and $k T$ has the usual meaning. The free energy of activation from the starting state to the transition state is $\Delta G^{*}=\Delta E^{*}+P \Delta V^{*}-T \Delta S^{*}$, where $\Delta S^{*}$ is the activation entropy, $\Delta V^{*}$ is the activation volume, and $P$ is the pressure. For SPEG in Si and Ge, $-\Delta G^{\mathrm{o}} / k T \gg 1$ and the final factor in eq. (1) can be dropped, yielding

$$
\mathrm{v}=A \lambda v \exp \left(-\frac{\Delta G^{*}}{k T}\right)
$$


Temperature-dependence experiments at zero or ambient pressure reveal the activation energy, given by $\Delta E^{*}=-k \partial(\ln \mathrm{v}) / \partial(1 / T)$, while isothermal pressure-dependence experiments reveal the activation volume, given by $\Delta V^{*}=-k T \partial(\ln v) / \partial P$. (Here the slight temperature and pressure dependence of $A, v$, and $\lambda$ are ignored.) Measured values of the prefactor and both $\Delta E^{*}$ and $\Delta V^{*}$ must be accounted for by any successful kinetic model. For a process controlled by the transport of a point defect present in equilibrium concentrations, $\Delta V^{*}$ is in many cases given by the sum of $\Delta V_{\mathrm{f}}^{O}$ (the formation volume of the defect) and $\Delta V_{\mathrm{m}}^{O}$ (the volume of motion of the defect).

Recently we reported that the SPEG rates in undoped $\mathrm{Si}^{22}$ and $\mathrm{Ge}^{23}$ are enhanced by pressure and can be characterized by negative activation volumes of $-0.28 \Omega \mathrm{Si}$ and $-0.45 \Omega \mathrm{Ge}$ respectively, where $\Omega$ is the crystalline atomic volume. These measurements allowed us to draw conclusions $^{23,24}$ about the plausibility of some of the models listed in Table I. Here we present in detail measurements of pressure-enhanced SPEG in undoped Si and Ge and measurements of the combined effects of pressure and doping on the SPEG rate in Si. We also report results of a search for the effects of amorphous-phase relaxation on the SPEG rate. All of the models are discussed in light of our measurements and of several kinetic arguments offered. Conclusions on the plausibility of the various types of models are presented. A kinetic analysis of the most plausible model is presented and a comparison with experiment is made. Finally, changes in the SPEG rate due to doping and ion irradiation are discussed.

\section{EXPERIMENT}

\subsection{Sample Preparation}

Wafers of single crystal (100)-oriented $\mathrm{Si}$ (p type, 1-2 $\Omega \mathrm{cm}$ ) and intrinsic (100)-oriented Ge were polished on both sides to a thickness of $25-40 \mu \mathrm{m}$. For the Si wafers, a double ion-implantation at $77 \mathrm{~K}$ with ${ }^{30} \mathrm{Si}^{+}$at $180 \mathrm{keV}, 2 \times 10^{15} / \mathrm{cm}^{2}, 0.4 \mu \mathrm{A} / \mathrm{cm}^{2}$ and at $60 \mathrm{keV}$, $1 \mathrm{x} 10^{15} / \mathrm{cm}^{2}, 0.4 \mu \mathrm{A} / \mathrm{cm}^{2}$ was used to produce a uniform amorphous layer approximately $300 \mathrm{~nm}$ thick. On some of them both sides were amorphized with the double implantation. For all the Ge wafers, only one side was amorphized at $77 \mathrm{~K}$ by a double ion implantation with ${ }^{74} \mathrm{Ge}^{+}$at 150 $\mathrm{keV}, 3 \times 10^{15} / \mathrm{cm}^{2}, 2 \mu \mathrm{A} / \mathrm{cm}^{2}$ and with a mixture of Ge isotopes at $1 \mathrm{MeV}, 5 \times 10^{15} / \mathrm{cm}^{2}, 140$ $\mathrm{n} A / \mathrm{cm}^{2}$. An amorphous Ge layer approximately $800 \mathrm{~nm}$ thick was determined by Rutherford backscattering and ion channeling. Dopant species As, B, or $\mathrm{P}$ were introduced by ion implantation into some pre-amorphized Si wafers. The As profile was implanted at room temperature with ${ }^{75} \mathrm{As}^{+}$at $75 \mathrm{keV}, 1 \times 1015 / \mathrm{cm}^{2}, 2 \mu \mathrm{A} / \mathrm{cm}^{2}$, and the $\mathrm{B}$ and $\mathrm{P}$ profiles were implanted at $77 \mathrm{~K}$ with ${ }^{11} \mathrm{~B}^{+}$at $35 \mathrm{keV}, 1.5 \times 10^{15} / \mathrm{cm}^{2}$ and ${ }^{31} \mathrm{P}^{+}$at $110 \mathrm{keV}, 1.5 \times 10^{15} / \mathrm{cm}^{2}$, respectively. To generate compensated samples, we used overlapping implantation of both B and $\mathrm{P}$ with the respective energies and doses given above; implantation energies were chosen for peak concentrations below 0.5 at $\%$ to avoid precipitation ${ }^{3}$ and for peaks situated no more than $200 \mathrm{~nm}$ beneath the surface, which is about the depth limit for accurate determination of the growth rate (see below, section $\mathbf{2 . 3}$ ).

To distinguish it from the implanted side of wafers prepared on one side only, the unimplanted side was sputter-coated with a thin $\mathrm{Al}_{2} \mathrm{O}_{3}$ layer ${ }^{25}$ at high oxygen partial pressure $\left(3 \times 10^{-4}\right.$ torr) with a pure aluminum target, a thickness of roughly $40 \mathrm{~nm}$ coloring the Si wafer red 
and the Ge blue. The stoichiometry of the oxide film was confirmed by x-ray photoelectron spectrometry (XPS). The wafers were then diced or cleaved into many small pieces (about 250 $\mu \mathrm{m} \times 250 \mu \mathrm{m})$ for high pressure treatment.

\subsection{High-Pressure Apparatus}

Samples were crystallized in a high-temperature and high-pressure diamond anvil cell (DAC) of the Piermarini-Block design ${ }^{26}$ with added external heating geometry, as shown in Fig. 1(a). (For a review of the DAC see Jayaraman ${ }^{27}$.) A detailed description of the cell and the techniques used for the present study can be found in 28 . Figure 1(b) is an expanded sketch of the pressure chamber of the DAC. The entire cell, from main body to adjusting screws, was made of the precipitation-hardened nickel-based alloy Udimet 718. The anvils were natural type-I diamonds, 8 -sided or 16-sided standard cut, polished with culets between $0.6 \mathrm{~mm}$ and $1.0 \mathrm{~mm}$ in size. Metal gaskets made of 0.01-in.-thick Inconel X750 sheet were pre-indented with the diamonds; holes about $350 \mu \mathrm{m}$ in diameter were subsequently drilled in the center of the indentation. After a sample and a small $(5-10 \mu \mathrm{m})$ ruby chip were placed into the gasket hole, the cell was cooled to liquid-nitrogen temperature while gaseous argon flowed across the diamond anvils. Throughout cooling, the gasket hole was monitored with an optical microscope. The chamber was sealed after the gasket hole was completely filled with liquid argon.

We used the ruby fluorescence technique 29 , which measures pressure-induced wavelength shifts of the ruby fluorescence lines $\left(\mathrm{R}_{2}: 692.7 \mathrm{~nm}, \mathrm{R}_{1}: 694.2 \mathrm{~nm}\right)$ excited by a HeCd laser $(441$ $\mathrm{nm})$, to measure the pressure on the sample. The pressure-induced shifts in both lines is 0.365 $\mathrm{nm} / \mathrm{GPa}$. Figure 2 is a schematic of the optical system used to measure the pressure. The wavelength of a neon lamp with a strong spectral line at about $693 \mathrm{~nm}$ served as a reference for the spectrometer. Because at high temperatures $\left(>300^{\circ} \mathrm{C}\right)$ the two ruby fluorescence lines weaken and broaden too much to permit a pressure determination, our pressure measurements were made at room temperature. In order to determine how the pressure might change upon heating, a simple calculation based on the thermal expansion of the argon pressure medium has been made 28 for the worst possible situation, i.e. assuming the volume of the sample chamber is fixed during heating. Using the equation of state 30 and the melting curve ${ }^{31}$ of argon, we find for the worst case that the pressure increases by $\sim 43 \%$ with an increase from room temperature to $550^{\circ} \mathrm{C}$, with most of the increase (about 60\%) coming from melting of the solid argon medium. Nevertheless, a simple experiment showed very little pressure change on the sample by heating up the cell to a temperature beyond the argon melting point. A cell with a pressure of $2.2 \mathrm{GPa}(22 \mathrm{kbar})$ at room temperature

was heated to $140^{\circ} \mathrm{C}$ (i.e., beyond the melting temperature of $\sim 120^{\circ} \mathrm{C}$ of $\operatorname{argon}^{31}$ at this pressure), and, using the ruby fluorescence technique, the pressure was measured again. After correction for the ruby line shift with temperature, which occurs independent of pressure ${ }^{32}$, the pressure at $140^{\circ} \mathrm{C}$ was unchanged within the experimental error $( \pm 0.15 \mathrm{GPa})$. Therefore we believe that the change in pressure upon heating from room temperature to $550^{\circ} \mathrm{C}$ cannot exceed $19 \%$, and, given that the load on the diamond anvils is held constant by Belleville spring washers which remain cool during the entire run, it is probably even lower. Others ${ }^{33,34}$ have seen much smaller changes in the same $\mathrm{P}-\mathrm{T}$ region.

The cell was heated by flexible resistive wire coiled around the pressure chamber. The power to the heater was regulated by a simple TECO (model TC-1000) controller. During heating, the cell was well insulated using $\mathrm{ZrO}_{2}$ felt in order to maintain temperature uniformity. A chromel-alumel thermocouple, placed next to the diamond and in contact with the metal gasket, was used to monitor the temperature. During the course of an annealing run, the temperature in the 
cell was maintained at $\pm 1^{\circ} \mathrm{C}$, but the temperature of the sample was lower than the thermocouple reading, as was determined by measuring the SPEG rate in the cell at atmospheric pressure. Using this as a calibration, we estimate 28 the accuracy in the sample temperature determination to be better than $\pm 5^{\circ} \mathrm{C}$.

\subsection{Optical Interferometry System}

The technique of in situ optical interferometry, or time-resolved reflectivity (TRR), developed by Olson et al. ${ }^{35}$ was used to monitor the moving c/a interface and then calculate the solid phase epitaxial growth rate in both $\mathrm{Si}$ and $\mathrm{Ge}$. Alternating constructive and destructive interference signals of laser reflections from the free surface and the c/a interface are detected by a photodiode and recorded. Figure 3 is a schematic of the optical system used to measure $\mathrm{Si}$ and $\mathrm{Ge}$ $\mathrm{SPEG}$ rates in the diamond anvil cell. For $\mathrm{Si}$, a single line $\mathrm{HeNe}$ laser $(5 \mathrm{~mW}, \lambda=633 \mathrm{~nm})$ and $\mathrm{Si}$ photodiodes were used. The refractive index of amorphous $\mathrm{Si}, n$, at this wavelength at $550^{\circ} \mathrm{C}$ is about $4.80^{3}$. Peaks in the TRR trace occur every time the interface moves a distance of $\lambda / 2 n$, or $65.9 \mathrm{~nm}$.

An infrared probe laser was necessary to make the optical interferometry technique useful in Ge due to its large absorption coefficient in the visible. We used a two-line HeNe laser source [red: $2 \mathrm{~mW}, \lambda=633 \mathrm{~nm}$; and infrared (IR): $0.5 \mathrm{~mW}, \lambda=1162 \mathrm{~nm}$ ] manufactured by Particle Measuring Systems (PMS). The visible red line enabled us to locate and focus the laser onto the microscopic sample in the cell. The IR line used for interferometry was detected with a Ge photodiode. At $\lambda=1162 \mathrm{~nm}$, the refractive index of amorphous Ge at room temperature 36 is 4.70, for a peak-to-peak distance in the interference trace of about $123.6 \mathrm{~nm}$. For TRR of both $\mathrm{Si}$ and $\mathrm{Ge}$, a voltage divider was used to cancel noises from the laser source, as shown in Fig. 3.

\section{RESULTS}

\subsection{Pressure-Enhanced SPEG of Ge}

In Table II , we list annealing runs for the SPEG of Ge in the diamond anvil cell at four temperatures and at pressures up to $5.0 \mathrm{GPa}(50 \mathrm{kbar})$. The growth rates were obtained by constructing depth vs. time plots by combining measured TRR traces and a theoretical reflectivity

vs. amorphous thickness curve calculated at $\lambda=1162 \mathrm{~nm}^{28}$. The peak-to-trough distance is independent of depth if the absorption coefficient in the amorphous phase is a constant 28 . Figure 4 is a TRR trace from Ge SPEG and the growth rate determined from it by this method. The rate was constant over the range $500-50 \mathrm{~nm}$ but slowed when the c/a interface reached about $50 \mathrm{~nm}$ below the surface. We believe this rate-retardation effect near the surface may be due to impurities, e.g., O, N, C, or H present on the surface of the sample and bombarded into it during ion implantation. The presence of $\mathrm{O}, \mathrm{N}$ and $\mathrm{H}$ has been demonstrated to retard the growth velocity in $\mathrm{Si}^{37,38}$.

Figure 5, which shows the portions of the TRR traces with uniform growth rates obtained for various pressures, clearly indicates that pressure increases $\mathrm{V}$ in $\mathrm{Ge}$. The rate increases by a factor of 100 for every 4.2 GPa increase in pressure. Several runs were attempted at and beyond $6.0 \mathrm{GPa}$, but none resulted in interpretable TRR traces ${ }^{28}$. Shimomura et al. ${ }^{39}$ reported a semiconductor-to- metal structural transition at $\sim 6 \mathrm{GPa}$ in vacuum-evaporated a-Ge at room 
temperature, and we suspect this phase transition eliminated the TRR traces in the runs at highest pressure.

Figure 6 is a plot of the temperature dependence of $v$ in our ambient pressure results (anneals in the DAC without argon loading) along with the original data of Csepregi et al. $^{2}$. The activation energy $\left(\Delta E^{*}\right)$ and the prefactor $\left(\mathrm{V}_{0}\right)$ can be found from the slope and intercept of the Arrhenius line. We found $\Delta E^{*}=2.17 \pm 0.2 \mathrm{eV}$ and $\mathrm{v}_{0}=1.2 \times 10^{7} \mathrm{~m} / \mathrm{s}$. The activation energy is in good agreement with the value of $2.0 \mathrm{eV}$ reported by Csepregi et al., but growth rates are lower by a factor of $\sim 5$. The source of this discrepancy is not clear, but it may be in part because the growth rates reported by Csepregi et al. were for Ge amorphized by ${ }^{28} \mathrm{Si}^{+}$implantation, while our a-Ge was self-implanted. We calibrated the temperature in the DAC by comparing SPEG rates of intrinsic Ge with those obtained on a hot stage, on which the sample temperatures were calibrated using temperature indicating lacquers (Omega Engineering Corp.).

Figure 7 is an isothermal plot for the activation volume of the SPEG process in the temperature range 300 to $365^{\circ} \mathrm{C}$. We found an activation volume of $-6.3 \pm 0.6 \mathrm{~cm}^{3} / \mathrm{mol}$, which is about $-46 \%$ of $\Omega_{\mathrm{Ge}}$, and the value was approximately the same at all temperatures. (Owing to the pressure calibration used in the earliest measurements, but later improved, early data at $325^{\circ} \mathrm{C}$ are somewhat scattered, with large error bars on the earliest measurements ${ }^{28}$.)

The question arises whether the pressure-enhanced SPEG rates observed in this work were due to increased dislocation densities from a nonhydrostatic component in the pressure. If we assume that dislocations enhance the SPEG rate and that the dislocation density increases with applied pressure, then a simple experiment can be carried out to address this question. First, a partial anneal (incomplete crystallization of the sample) is performed at high pressure, then the pressure is lowered and the anneal on the same sample is finished. If rate-enhancement were due to an increased dislocation density at high pressures, the SPEG rates in a sample brought from a high to an intermediate pressure would differ from rates in a sample otherwise identical but not subjected to high pressure. We carried out an experiment of this type for SPEG in Ge. First, a partial anneal was performed at $300^{\circ} \mathrm{C}$ and $3.6 \mathrm{GPa}$, leaving an amorphous over layer $100 \mathrm{~nm}$ thick, and then the pressure was lowered to $1.8 \mathrm{GPa}$ and the sample annealed at a higher temperature, $325^{\circ} \mathrm{C}$, to complete growth. Both growth rates fell closely on their corresponding rate vs pressure isotherms, clearly indicating that $\mathrm{V}$ is not affected by pressure history. A frequent finding that supports this conclusion is that when, prior to an annealing run, the pressure was adjusted in pursuit of a specific value, the growth rates always fell on the same rate vs pressure isotherms independent of whether the pressure had been ever lowered to the final from a greater value. Taken together, our results indicate that enhancement of SPEG rates from pressure is due not to increases in dislocation densities with pressure but to the effect of pressure on the kinetics of growth, as characterized by the activation volume.

\subsection{Pressure-Enhanced SPEG of Undoped Si}

Table III lists all conditions of the runs and SPEG rates of Si. Figure 8 shows a $\lambda=633 \mathrm{~nm}$ TRR trace of a sample annealed at $520^{\circ} \mathrm{C}$ and $1.1 \mathrm{GPa}$, and the corresponding depth vs time curve. As in the other runs with these samples, the growth rate was constant throughout the amorphous thickness. Figure 9, an Arrhenius plot of our ambient pressure results (anneals in the DAC without argon loading), shows good agreement with the data of Olson et al. ${ }^{3}$ for similar material. Figure 10 demonstrates that in $\mathrm{Si}$, as in $\mathrm{Ge}$, hydrostatic pressure enhances $\mathrm{V}$, in this case by a 
factor of 5 for every $3.2 \mathrm{GPa}$.

Figure 11 is an activation volume plot of $\mathrm{V}$ in undoped $\mathrm{Si}$. The average value of $\Delta V^{*}$ is $-3.3 \pm 0.3 \mathrm{~cm}^{3} / \mathrm{mol}$, which is about $-28 \%$ of $\Omega_{\mathrm{Si}}$. This negative activation volume agrees qualitatively but not quantitatively with that of Nygren et al. ${ }^{40}$, who found a value of roughly $-70 \% \Omega_{\mathrm{Si}}$ for the same process. They used a piston cylinder apparatus with solid $\mathrm{NaCl}$ as the pressure transmission medium to generate pressures up to $2.0 \mathrm{GPa}$ and used Rutherford backscattering spectrometry (RBS) and ion-channeling techniques to measure the growth rates. A recent measurement of the effect of nonhydrostatic stresses on the SPEG rate ${ }^{41}$ suggests that their rates were probably enhanced beyond the hydrostatic values by an undetermined amount of uniaxial compression.

\subsection{Pressure-Enhanced SPEG of Doped Si}

Table IV lists conditions of the SPEG runs for each of four differently doped Si samples. The table does not include growth rates, which varied in time due to nonuniform dopant profiles. A demonstration that pressure enhances the growth rate is presented in Figure 12, a series of TRR traces from boron-doped samples at $520^{\circ} \mathrm{C}$; the dependence of $\mathrm{V}$ on dopant concentration is demonstrated by the varying intervals between peaks and troughs. To calculate the dependence of the growth rate on dopant concentration and pressure, experimental TRR traces are converted into a velocity vs interface depth curve with the following procedure 28 . For the time coordinate of each point in a peak-to-trough segment, we calculated the depth corresponding to the same scaled reflectivity value in the same segment of a theoretical reflectivity vs amorphous thickness curve. This procedure for mapping the experimental (reflectivity vs time) trace onto the theoretical (reflectivity vs amorphous thickness) curve is calibrated at every peak and trough of the TRR trace. By taking point-by-point derivatives of the depth-vs.-time curve we determined the growth rate vs depth.

Figure 13, growth rate vs depth curves for three B-doped Si samples annealed at three different pressures, shows the combined rate-enhancements of doping and pressure. At ambient pressure, the amount of enhancement due to the dopant is in good agreement with values reported by Park et al. ${ }^{42}$. The ratio of growth rates at high pressure to that at ambient pressure at the same depth is plotted against the right-hand axis. The velocity ratio, a measure of the pressure effect, depends only weakly, if at all, on either depth or dopant concentration.

Results similar to those shown in Figures 12 and 13 were obtained 28 with other types of doped samples, with ambient pressure results in good agreement with previously published values $^{3,43}$. The growth rate of the sample with both boron and phosphorus dopants exhibited, to a large extent, the compensation effect ${ }^{3}$ : the SPEG rate in compensated samples is reduced to that in undoped samples. An exact compensation was not obtained because of the single-energy nature of each implantation. Depth profiling of compensated samples ${ }^{28}$ by Secondary Ion Mass Spectrometry (SIMS) revealed a mismatch in the positions of the peaks of the $\mathrm{B}$ and $\mathrm{P}$ concentrations, but the ratio of the rate at high pressure to that at ambient pressure in all samples confirms the weak or negligible dependence of pressure-enhancement on dopant concentration. The activation volume of doped and compensated samples, shown in Figure 14, is indistinguishable from that of undoped Si shown in Figure 11. That all points in Fig. 14 lie on the same line indicates the independence of activation volume on dopant type. Our measurements probably lack the precision to find dopant-induced changes in $\Delta \mathrm{V}^{*}$ if they are on the order of $10 \%$, as is seen in $\Delta \mathrm{E}^{*}$ of doped samples 42,43 .

\subsection{Search for an Effect on SPEG of Amorphous Relaxation}


Studies of annealed a-Si and a-Ge $\mathrm{G}^{5,44-46}$ have revealed a pre-crystallization exothermic process believed to come from structural relaxation of the amorphous phase. The heats of relaxation in both a-Si and a-Ge have been measured using differential scanning calorimetry (DSC). In Si, the heat is about $36 \%$ of the heat of crystallization from the DSC curve 44 reproduced in Figure 15. Two sources for the physical origin of the relaxation process have been suggested: (1) local atomic readjustments that relieve large bond-angle distortions ${ }^{47}$ frozen in during the formation of the amorphous phase; and (2) the annihilation of point defects remaining in the amorphous phase ${ }^{44-46}$. On the basis of spectroscopic studies ${ }^{48}$, the two point defects that have been proposed are dangling bonds 48 (threefold-coordinated atoms) and floating bonds 49 (fivefold-coordinated atoms); both are analogous to the two predominant point defects in crystals (vacancies and interstitials). Waddell et al. ${ }^{50}$ have shown that for self-implanted a-Si, the concentration of spectroscopic defects decreases during structural relaxation by about a factor of 2 from $2 \times 10^{19} \mathrm{~cm}^{-3}$ after annealing at $500^{\circ} \mathrm{C}$.

Structural relaxation affects atomic diffusion in amorphous systems $51-53$. Typically, the diffusion coefficient in an amorphous phase is higher in the unrelaxed than in the relaxed state. In a study of interdiffusion in a-Si/a-Ge multilayers (Figure 16) Park et al. ${ }^{52}$ found that the diffusivity in the unrelaxed state was higher than in the relaxed state by a factor of $\sim 5-10$. Correspondingly, we searched for an effect of amorphous relaxation on the SPEG rate in both Si and $\mathrm{Ge}$ from an examination of its time dependence.

Wafers of (100)-oriented single-crystal Si (p-type, $1-2 \mathrm{k} \Omega \mathrm{cm}, 0.4 \mathrm{~mm}$ thick) were polished on one side and dual-implantation-amorphized at $77 \mathrm{~K}$ with $\sim 10 \mu \mathrm{A} / \mathrm{cm}^{2}$ of ${ }^{30} \mathrm{Si}^{+}$at $180 \mathrm{keV}$ to a dose of $2 \times 10^{15} / \mathrm{cm}^{2}$, and $60 \mathrm{keV}$ to doses of $1 \times 10^{15} / \mathrm{cm}^{2}$ or $5 \times 10^{15} / \mathrm{cm}^{2}$. Wafers of (100)-oriented single-crystal Ge (intrinsic, $\sim 1 \mathrm{~mm}$ thick) polished on one side were dualimplantation-amorphized at $77 \mathrm{~K}$ with $4 \mu \mathrm{A} / \mathrm{cm}^{2}$ of $76 \mathrm{Ge}^{+}$at $180 \mathrm{keV}$ to a dose of $2 \times 10^{16} / \mathrm{cm}^{2}$, and at $75 \mathrm{keV}$ to a dose of $5 \times 10^{15} / \mathrm{cm}^{2}$. To create a deeper amorphous layer, some Ge wafers were further implanted at $77 \mathrm{~K}$ with $\mathrm{Ge}^{+}$of natural isotopic abundance at $1 \mathrm{MeV}$ to a dose of $5 \times 10^{15} / \mathrm{cm}^{2}$. All wafers were diced into $1 \mathrm{~mm} \times 1 \mathrm{~mm}$ or $2 \mathrm{~mm} \times 2 \mathrm{~mm}$ samples.

SPEG anneals of the Si and Ge samples were carried out in air on a cylindrical hot stage consisting of a nickel rod $1.90 \mathrm{~cm}$ in diameter in contact with heating coil windings. After the heater block had stabilized at a desired annealing temperature, a sample was quickly placed on top of it. Intervals between initial contact of the sample with the hot stage and the beginning of data collection were typically $5-10 \mathrm{sec}$. The accuracy of the temperature calibration is discussed by $\mathrm{Lu}^{28}$. The in situ TRR technique was used to obtain the instantaneous velocity of the c/a interface. For SPEG in Si samples, both a HeNe $633 \mathrm{~nm}$ laser and an infrared light-emitting diode (LED) with a peak wavelength at $935 \mathrm{~nm}$ and $50 \mathrm{~nm}$ FWHM were used to probe the sample surface. At the longer wavelength the a-Si absorption coefficient is reduced, greatly improving the sensitivity of the TRR technique for monitoring the c/a interface at greater depth; however, owing to the light source some depth resolution is lost. For SPEG in Ge samples only the infrared LED was used. At this wavelength, the refractive index $\mathrm{n}$ for a-Ge is ${ }^{36}$ about 4.85 ; it is about 4.80 for a-Si (determined by comparing TRR at 935 and $633 \mathrm{~nm}^{28}$ ).

Figure 17 shows TRR traces from two Si samples crystallized on the hot stage at $630^{\circ} \mathrm{C}$ and the derived growth rate vs time curves. Both traces were obtained using the infrared LED. One sample was pre-annealed at $523^{\circ} \mathrm{C}$ for about 9 minutes and then placed on the heater block for SPEG. This pre-annealing treatment almost completely relaxes the a-Si, according to the 
spectroscopy results of Waddell et al. ${ }^{50}$ and the calorimetry results of Donovan et al. ${ }^{44}$ (note time axis in Fig. 15). The time constant for relaxation derived from the calorimetry work remains in the range 200-350 sec at the pre-anneal temperature and at the temperature of SPEG ${ }^{44}$; hence there was sufficient time to relax the sample during the pre-anneal but insufficient time at the temperature of SPEG to bring both samples in Fig. 17 to the same structural state. SPEG runs were performed by placing the sample on a pre-heated block, the sample reaching its final SPEG temperature within seconds. The figure indicates no significant difference in growth behavior between relaxed and unrelaxed samples, a finding that was confirmed in other samples of $\mathrm{Si}$ and of $\mathrm{Ge}$ as well 28 . For both samples in Fig. 17, the growth rate rapidly decays to a steady-state value of about $60 \%$ of the initial value, a phenomenon generally observed for $\mathrm{Si}$ in all of more than 20 other SPEG runs at temperatures ranging from $540^{\circ} \mathrm{C}$ to $690^{\circ} \mathrm{C}$. In Ge, the steady-state value was about $40 \%$ of the initial value.

The observation of rapid decay in the SPEG rate in both Si and Ge might at first glance be interpreted as an effect of structural relaxation. The magnitude of the steady-state value in $\mathrm{Si}$, however, is about $60 \%$ of the initial value, which compares poorly with the $20 \%$ ratio found in Park's diffusion study 52 . That the initial decay is independent of the growth temperature, of amorphous layer thickness (studied in Ge only), and, most important, of the thermal history of the samples suggests that the initial decay observed in both $\mathrm{Si}$ and $\mathrm{Ge}$ for the most part is not due to structural relaxation. Its most likely source is atomic hydrogen, as recently discovered by Roth and Olson ${ }^{38}$. The implications of the lack of a structural relaxation effect on $v$ for the growth mechanism are discussed below (Section 4.5).

\section{DiscuSSION}

Although the SPEG process occurs by motion of a sharp c/a interface, models conflict on whether the process is controlled by interface reaction kinetics 12,13 or by bulk diffusion of defects to the interface $2,6,14-17$. We address both possibilities in the discussion that follows.

\subsection{Implications for Bulk Point Defect Mechanisms}

For any bulk point-defect mechanism of thermal SPEG in which defect impingement on the interface acts in series with atomic reaction at the interface, the growth rate can be expressed by

$$
\mathrm{v} \propto \frac{\mathrm{k}_{\mathrm{d}} \cdot \mathrm{k}_{\mathrm{i}}}{\mathrm{k}_{\mathrm{d}}+\mathrm{k}_{\mathrm{i}}}
$$

where $\mathrm{k}_{\mathrm{d}}$ is the rate of the bulk generation and transport of defects to the interface and $\mathrm{k}_{\mathrm{i}}$ is the rate of reaction at the interface. In general, both $\mathrm{k}_{\mathrm{d}}$ and $\mathrm{k}_{\mathrm{i}}$ have Arrhenius form. Their product is expected to have Arrhenius form if one of these rate constants is negligibly small with respect to the other (the unlikely alternative is that they have identical activation energies - and volumes). In SPEG of Si, the single activation energy over 10 decades in velocity ${ }^{3}$, as shown in Fig. 9, offers strong evidence for a single significantly rate-limiting step. If the limiting step is the interface reaction then it is not really a bulk defect mechanism, in which case how defects are transported to the interface becomes almost as peripheral a question as how thermal energy is transported to the atoms in the reaction. If, however, the rate-limiting step is bulk defect generation and transport to the interface, then the SPEG rate and bulk diffusivity can be directly compared. For both processes, the rate would be given by a product of the concentration of point defects, their mobility, and a geometrical factor. Hence, 


$$
\frac{\mathrm{v}}{\text { Dbulk }}=\text { constant }
$$

Furthermore, a long-range-diffusion limited crystal growth process would normally be expected to yield parabolic growth kinetics. With the exception of the initial transient, which appears to be caused by hydrogen ${ }^{38}$, the SPEG runs in this and other studies yielded timeindependent velocities. This result alone does not rule out bulk diffusion as the rate-limiting step in SPEG, but an unusual bulk diffusion model would be needed to account for the constant velocity.

\subsection{Implications for Vacancies in Ge}

Suni et al. ${ }^{6}$ adopted the suggestion of Csepregi et al. ${ }^{2}$ and proposed that the rate-limiting step is the formation and migration of lattice vacancies to the c/a interface. Letaw et al. ${ }^{54}$ have suggested that crystal vacancies are the point defects responsible for another important and wellstudied kinetic process, namely, atomic diffusion in crystalline $\mathrm{Ge}$, a suggestion strongly supported by diffusion studies ${ }^{55-59}$. Recently, Werner et al. ${ }^{60}$ produced direct evidence for the vacancy mechanism of diffusion when they found a positive activation volume in their highpressure study of Ge self-diffusion. They measured the tracer-diffusion coefficient for ${ }^{71} \mathrm{Ge}$ as a function of pressure, temperature, and doping and found that hydrostatic pressure reduces the diffusivity and that n-type doping enhances diffusion and p-type doping retards it. The doping behavior demonstrates that the vacancy has an acceptor level or a negatively charged state in the band gap. On the basis of their data, Werner et al. deduced $+0.56 \Omega_{\mathrm{Ge}}$ for the activation volume of diffusion by the neutral vacancy and $+0.28 \Omega_{\mathrm{Ge}}$ by the negatively charged vacancy. The clear difference between that value and the negative activation volume of $-0.46 \Omega_{\mathrm{Ge}}$ obtained in our study of SPEG in Ge shows that Eq. (4) is violated, hence the defects that limit self-diffusion in the crystal and those limiting the SPEG rate are not the same.

\subsection{Implications for Vacancies in $\mathrm{Si}$}

Despite extensive research on diffusion in $\mathrm{Si}$, whether the diffusion process is mediated by interstitials or vacancies remains open. In this laboratory attempts have been made to study the effect of pressure on Si self-diffusion, as Werner et al. ${ }^{60} \mathrm{did}$ for Ge, and in preliminary results Aziz et al. ${ }^{61}$ reported qualitative results showing pressure-enhanced $S i$ self-diffusion. Owing to experimental difficulties in pressure generation with a solid pressure-transmission medium, the measurement was not deemed sufficiently reliable to make or break models, and the problem continues under investigation. As a result, no direct comparison of the activation volume of diffusion by the vacancy mechanism and that of SPEG can be made for $\mathrm{Si}$, as was possible for $\mathrm{Ge}$ above. There have been, however, several theoretical studies ${ }^{62-66}$ of the formation volume of a vacancy in crystalline $\mathrm{Si}$. In an early study by Larkins and Stoneham ${ }^{62}$, calculation of the lattice relaxation around a vacancy resulted in a value of $78 \%$ of $\Omega_{\mathrm{Si}}$ for the formation volume of a neutral vacancy. A recent total energy calculation by Antonelli and Bernholc 66 of pressure effects on Si self-diffusion yielded a vacancy formation volume of $75 \%$ of $\Omega \mathrm{Si}$. Here we relate the theoretical formation volume to the activation volume measured for SPEG in Si and discuss the implications for the vacancy mechanism of SPEG.

If SPEG in Si were controlled by the diffusion of vacancies in the crystal, the observed pressure-enhanced SPEG would necessarily be due to pressure reducing the barrier to vacancy motion enough to overwhelm the pressure-reduced vacancy concentration. In this case, an upper limit can be placed on the formation volume of the vacancy because the barrier to motion cannot be 
reduced past zero without something dramatic occurring, e.g., the slope of the $\mathrm{V}$ vs. pressure isotherm might change sign. The barrier for vacancy migration $\Delta E_{\mathrm{m}}^{*}$, according to Watkins ${ }^{67}$, is $0.45 \mathrm{eV}$ for neutral vacancies.

No breaks were observed up to the highest pressure-annealing point, $P_{\max }=5.0 \mathrm{GPa}$ at $520^{\circ} \mathrm{C}$, in Figure 11 . By requiring

$$
\Delta E_{\mathrm{m}}^{*}+P_{\max } \Delta V_{\mathrm{m}}^{*} \geq 0
$$

we obtain a lower limit for the migration volume of a vacancy: $\Delta V_{\mathrm{m}}^{*} \geq-0.72 \Omega_{\mathrm{Si}}$. Since the measured $\Delta \mathrm{V}^{*}$ for SPEG $\left(-0.28 \Omega_{\mathrm{Si}}\right)$ is the sum of $\Delta V_{\mathrm{m}}^{*}$ and the formation volume $\Delta V_{f}^{0}$, an upper limit on the formation volume is given by

$$
\Delta V_{f}^{0}<+0.44 \Omega \mathrm{Si}
$$

This is much smaller than all the theoretical values of which we are aware. We therefore consider it highly implausible that in Si the formation and transport of crystal vacancies to the c/a interface is rate-limiting step for the SPEG process.

\subsection{Implications for Minority Diffusers}

Further considerations exist for any mechanism that involves point defects from the bulk of either phase and in which the defects contribute even a small amount to self diffusion. By comparing $\mathrm{V}$ to the measured self-diffusivity, one can determine whether there are enough defects or whether they are mobile enough - to cause the measured SPEG rate. If each such defect converts $\mathrm{N}_{\mathrm{r}}$ atoms from the amorphous to the crystalline phase on arrival at the interface, $\mathrm{V}$ of (100)-oriented $\mathrm{Si}$ or Ge will be the product of the fraction of a monolayer crystallized per second and the spacing between monolayers:

$$
\mathrm{v}=\left(\frac{1}{2} \Gamma_{\mathrm{D}} \cdot \frac{C_{c / a}^{D} \cdot N_{r}}{C_{O}}\right) \cdot\left(\frac{a}{4}\right),
$$

where $\Gamma_{\mathrm{D}}$ is the jump frequency of the defect, $C_{\mathrm{c} / \mathrm{a}}$ the defect concentration at the c/a interface, $C_{O}$ the atomic concentration $(1 / \Omega)$, $a$ the crystal lattice parameter (equal to $4 / \sqrt{3}$ times the interatomic spacing $\lambda$ ), and the factor $1 / 2$ appears because only half the jumps move a vacancy toward the interface.

If self diffusion occurs by a number of point defect mechanisms operating in parallel, we can write the contribution to the self-diffusivity from any particular species of defect, $D_{D}^{S e l f}$, as

$$
D_{D}^{\text {Self }}=\frac{1}{6} \Gamma d^{2}
$$

where $\Gamma$ and $d$ are the average number of jumps that each atom makes per second and the average distance that an atom jumps, respectively, due to the particular defect mechanism under consideration. Assuming that one atom jumps per defect jump, we have 


$$
C_{O} \Gamma=C_{\mathrm{bulk}}^{D} \Gamma_{\mathrm{D}}
$$

where $C_{\mathrm{bulk}}^{D}$ is the bulk defect concentration. Combining (8) and (9),

$$
\Gamma_{\mathrm{D}}=\frac{6 D_{D}^{\text {self }} C_{O}}{d^{2} C_{\mathrm{bulk}}^{D}}
$$

Inserting into (7), we have

$$
\mathrm{v}=\frac{\sqrt{3} N_{r} \lambda D_{D}^{\text {self }} C_{c / a}^{D}}{d^{2} C_{\mathrm{bulk}}^{D}}
$$

Since the interface is acting as a sink for defects, $C_{c / a}^{D} \leq C_{\mathrm{bulk}}{ }_{\text {. Furthermore, the actual self- }}$ diffusion coefficient, $D^{\text {self }}$, will never be less than the contribution $D_{D}^{\text {self }}$ from the particular defect under consideration. Combining these inequalities with eq. (11) then places an upper limit on $\mathrm{V}$, which may be inverted to place a lower limit on $N_{r}$ :

$$
\mathrm{N}_{\mathrm{r}} \geq \frac{s^{2} \lambda}{\sqrt{ } 3} \frac{\mathrm{v}}{\mathrm{D}^{\text {self }}},
$$

where $s \equiv d / \lambda$. For crystal vacancies $s=1$. For an interstitial diffusion mechanism $s=(8 / 3)^{1 / 2}$; for an interstitialcy mechanism $s=2 / \sqrt{3}$. We assume that for vacancy-like and interstitial-like defects in the amorphous phase, $s \geq 1$. For dangling bonds and floating bonds in the amorphous phase, however, it is likely that $s<1$. Assuming the amount of diffusion to be a function only of the number of nearest neighbors that change when dangling bonds or vacancies migrate, we can estimate the magnitude of $s$ for dangling bonds. As is evident from Table $\mathrm{V}$, it takes $3 m$ jumps of a dangling bond to cause the same amount of diffusion as do $m$ jumps of a vacancy. Hence,

$$
\frac{1}{6}(3 m) d^{2}=\frac{1}{6} m \lambda^{2}
$$

or, for dangling bonds, $s=1 / \sqrt{3}$. Similar results are obtained for floating bonds. If eqs. (7)-(12) are modified to allow the correlation factors $f_{\mathrm{a}}$ for the diffusion of atoms and $f_{\mathrm{d}}$ for the diffusion of point defects to deviate from unity, the result is that the r.h.s. of eq. (12) is multiplied by $f_{\mathrm{a}} / f_{\mathrm{d}}$, which we neglect because we expect it to be very near unity.

The lower limit on $\mathrm{N}_{\mathrm{r}}$ is obtained from equation (12) using measured values of $\mathrm{v}$ and $\mathrm{D}^{\text {self. }}$. The self-diffusion coefficients in crystalline $\mathrm{Si}$ and $\mathrm{Ge}$ have been measured, although discrepancies persist in the temperature dependence for $\mathrm{Si}^{68}$. The data for self-diffusion in $\mathrm{Si}^{59}$ and $\mathrm{Ge}^{58}$ measured to low temperatures (1128 K in Si and $808 \mathrm{~K}$ in $\mathrm{Ge}$ ) are fitted by Arrhenius expressions with prefactors of 154 and $13.6 \mathrm{~cm}^{2} / \mathrm{sec}$, and activation energies of 4.65 and $3.09 \mathrm{eV}$, in $\mathrm{Si}$ and Ge respectively.

Although no direct measurements of self-diffusion coefficients in amorphous Si and Ge are available, values can be estimated by extrapolating the measurements of Park ${ }^{52}$ of the 
composition-dependent interdiffusion coefficient of a-Ge/a-Si multilayers to the pure Si or Ge limit (see Fig. 16). Strictly speaking, the extrapolations yield the tracer diffusivities of Si in a-Ge and $\mathrm{Ge}$ in a-Si but not the respective self-diffusivities. We expect the differences to be small because $\mathrm{Si}$ and $\mathrm{Ge}$ are chemically similar and form continuous solid solutions; this expectation is borne out in crystalline $\mathrm{Si} 68$.

At the equiatomic composition, Park also measured the prefactor and activation energy for the interdiffusivity to be $3.0 \times 10^{-3} \mathrm{~cm}^{2} / \mathrm{sec}$ and $2.34 \mathrm{eV}$, respectively. On the assumption that the prefactor does not depend on composition but that the activation energy does, the activation energies for his other two compositions, $25 \% \mathrm{Si}$ and $75 \% \mathrm{Si}$, were calculated from the data. Estimates of self diffusion coefficients for pure a-Si and a-Ge at any temperature were then obtained by (1) finding the interdiffusion coefficients at the temperature of interest for the three compositions, using the prefactor and the corresponding activation energies, and (2) extrapolating in composition to the pure limits.

Table VI lists estimates of lower limits for $\mathrm{N}_{\mathrm{r}}$ for the various types of defects proposed for Si and Ge SPEG. These results are based on only two major assumptions: that bulk diffusion of the defect controls the SPEG rate and that defect diffusion contributes to bulk self diffusion.

Among the estimates of the defect density in a-Si, the lowest is $\sim 10^{19} / \mathrm{cm}^{3}$ from the spectroscopy work of Waddell et al. ${ }^{50}$ in a fully relaxed amorphous phase formed by implantation. Other estimates are as high as a few atomic percent ${ }^{46}$. Since the lowest value corresponds to about 1 defect in every $10^{4}$ atoms, values of $\mathrm{N}_{\mathrm{r}}$ much above $10^{4}$ seem highly implausible, as we would expect the multiple rearrangement process to be interrupted upon encountering a defect. Therefore from the lower limits of $\mathrm{N}_{\mathrm{r}}$ determined above, we can draw the following conclusions about the possibilities of the various defect types whose diffusion to the c/a interface is postulated to be the rate limiting step for SPEG process. (1) It is highly implausible that in either c-Si or c-Ge, vacancies or self-interstitials are defects whose diffusion to the interface controls the SPEG rate. This follows simply because self diffusivities in c-Si and c-Ge are far too low to account for the observed SPEG rates. (2) Similarly, it is unlikely that in a-Ge the diffusion of vacancy-like and interstitial-like defects to the c/a interface controls the growth process. (3) This argument does not rule out dangling or floating bonds in both bulk a-Ge and a-Si, or the interstitial-like defect in a-Si, as the defects whose diffusion to the c/a interface controls SPEG.

\subsection{Implications of the Lack of Relaxation in SPEG Rate}

The relaxation experiment reported in section 3.4 indicated the absence of a significant effect of amorphous relaxation on the SPEG process in both Si and Ge. Although both materials exhibited a decay from initial to steady-state values by factors of 0.6 in Si and 0.4 in Ge, the independence of these factors from the thermal history of the samples strongly suggests that structural relaxation of the amorphous phase has little, if any, effect on SPEG. The absence of a relaxation effect distinguishes crystal growth from diffusion in the bulk of the amorphous phase, where large reductions in diffusivity by a factor of 0.1 to 0.2 were reported ${ }^{52}$ (see Fig. 16). We take this as evidence that different defects are responsible for SPEG and for diffusion.

The point defects considered in Table I can be categorized as residing primarily at the c/a interface or residing primarily in the bulk of one of the phases present. In section 4.4 we showed it highly implausible for any bulk point defect in either c-Si or c-Ge to be responsible for the SPEG process, although some bulk defect mechanisms from the amorphous phase were not ruled out. If we make the worst-case, implausible assumption that the entire SPEG relaxation effect is indeed due to a reduction in the concentration of bulk amorphous point defects that control SPEG, we can still use the observed difference in the relaxation effects on the growth and diffusion processes to 
set an upper limit on the contribution of the bulk defects in the amorphous phase. Assume that there are two processes occurring in parallel — an interfacial point defect process of constant rate and a bulk amorphous point defect process with a rate decaying to 0.2 of its initial value as does bulk diffusion - to produce the observed SPEG rate for both Si and Ge. It follows for Si that if the steady-state $\mathrm{V}$ is 0.6 of the initial $\mathrm{v}$, then the maximum fraction of the steady-state $\mathrm{V}$ that can be attributed to bulk point defects is $17 \%$. For Ge, the numbers are 0.4 and $38 \%$ respectively. With this argument we can conclude that no single defect in a-Si or a-Ge can be responsible for all of bulk diffusion in the amorphous phase and for a majority of solid phase epitaxy.

\subsection{Implications of the nonhydrostatic stress effect}

Recently, Aziz et al. ${ }^{41}$ found that the SPEG rate in Si is enhanced by uniaxial tension and reduced by uniaxial compression in a direction parallel to the surface, in contrast to the enhancement by hydrostatic pressure. The uniaxial and hydrostatic results were combined to determine the activation strain tensor for Si SPEG. Through an extension of transition state theory to nonhydrostatic stress states, the results were interpreted in terms of a "short and fat" transition state: locally, the system undergoes a contraction in the direction normal to the interface and an inplane expansion to reach the saddle-point configuration. [This interpretation has been confirmed in an independent experiment on samples under biaxial compression. by comparing SPEG rates on stressed and stress-relaxed substrates during heteroepitaxy of $\mathrm{Si}_{1-\mathrm{x}} \mathrm{Ge}_{\mathrm{X}}$ on $\mathrm{Si}$, Hong et al. (Q.-Z. Hong, J.G. Zhu, J.W. Mayer, W. Xia, and S.S. Lau, accepted for publication in J. Appl. Phys. [J.A.P. 71, 1768 (1992)]) find quantitative agreement with the value of the first element of the activation strain tensor reported in Ref. 41 by us.] The form of the activation strain tensor, describing a rate enhancement for hydrostatic compression and a reduction for uniaxial compression, is inconsistent with the formation or motion of any point defect in the bulk of the amorphous phase so long as the stress is fully relaxed, as measurements indicate 69 . With the stress fully relaxed, no change in point defect populations or mobilities should be observed in the amorphous phase. Even if some remnant of the applied uniaxial stress were to remain in the bulk of the amorphous phase, a uniaxial compression effect would be of the same sign but of smaller magnitude than the hydrostatic pressure effect. The c/a interface, with its reduced symmetry relative to that of the crystal, is the only place where the opposite-signed effects of uniaxial and hydrostatic compression can be reconciled. Hence the rate-limiting step for SPEG must occur at the interface.

\subsection{Implications of the orientation dependence}

The SPEG rate in Si varies by more than a factor of 20 as the orientation is varied, being fastest if the interface is oriented near (100) and slowest if it is oriented near (111). Since diffusion in both the cubic crystal and the amorphous phase is necessarily isotropic, this observation alone rules out pure bulk-diffusion-controlled mechanisms (see equation 4) and implies at least some significant role for interface kinetics in determining the overall SPEG rate. Some models combine bulk defect diffusion with interface attachment kinetics in limiting the overall rate. Any such combination would be inconsistent with the observed constant activation energy over 10 decades in growth rate, and with the observed constant activation volume over a more limited velocity range, unless the bulk diffusion process and the interfacial reaction process happen to have identical activation energies and volumes.

\subsection{Kinetic analysis of the dangling bond mechanism}

Taken together, the results make the Spaepen-Turnbull mechanism ${ }^{12}$, in which dangling bonds are generated at the interface and migrate along the interface reconstructing the random 
network into the crystalline network, one of the two proposed mechanisms that remain tenable. The other is the charged kink-site model of Williams and Elliman ${ }^{13}$, which will be discussed separately. The dangling-bond model in addition seems highly plausible because it predicted a negative activation volume ${ }^{70}$ : the mobility of dangling bonds is enhanced by pressure through a transition state with a lower local volume, in which the dangling bond reaches across a ring to attack a fully-coordinated $\mathrm{Si}$ or Ge. A kinetic analysis of the model was undertaken as a further test of its plausibility. Fig. 18 shows a reaction-coordinate diagram for the breaking, migration, and trapping of a dangling bond. A single bond breaks to form a pair of dangling bonds, each of which migrates independently, moving a net distance of $n_{r}$ migration steps and converting $m_{r}$ atoms from the amorphous to the crystal phase, before becoming annihilated or immobilized at one of a fixed number of "traps." In this analysis, $v$ is given by

$$
\mathrm{V}=F_{\mathrm{L}} P_{\mathrm{db}}<\mathrm{V}_{\mathrm{db}}>
$$

where $F_{\mathrm{L}}$ is the fraction of bonds at the interface that reside at a ledge, which is equal to the sine of the misorientation $\theta$ of the interface from (111), $P_{\mathrm{db}}$ is the probability that such a bond is dangling, and $\left\langle\mathrm{V}_{\mathrm{db}}\right\rangle$ is the average migration velocity of a dangling bond along the interface. If $N_{\mathrm{db}}$ and $N_{\mathrm{b}}$ are the number of dangling bonds and the total number of bonds per unit area of interface, respectively, then

$$
P_{\mathrm{db}}=\frac{N_{\mathrm{db}}}{N_{\mathrm{b}}} .
$$

The average velocity of a dangling bond is determined by the difference between its average forward and backward hopping rates, $k^{+}$and $k^{-}$respectively. Assuming that the dangling bond moves one nearest-neighbor distance with each hop,

$$
\left\langle\mathrm{v}_{\mathrm{db}}\right\rangle=\lambda\left(k^{+}-k^{-}\right)
$$

The average hopping rates of dangling bonds are obtained from transition-state theory:

$$
\begin{aligned}
& k^{+}=v \exp -\left(\frac{\Delta G_{\mathrm{m}}}{\mathrm{kT}}\right), \\
& k^{-}=v \exp -\left(\frac{\Delta G_{\mathrm{m}}-\Delta G^{\mathrm{o}}}{k T}\right),
\end{aligned}
$$

where $\Delta G_{\mathrm{m}}$ is the average barrier to dangling bond migration and $\Delta G^{\mathrm{o}}$ is the standard Gibbs free energy change per dangling bond migration event, defined to be negative for crystallization. As pointed out by Spaepen and Turnbull ${ }^{20}, \Delta G^{\mathrm{o}}$ is not necessarily equal to the free energy change per atom crystallized. If a dangling bond migration event transfers $r$ atoms from the amorphous phase to the crystal, $\Delta G^{\mathrm{o}}$ will be $r$ times the free energy change per atom crystallized. Spaepen's model ${ }^{71}$ of the (111) interface has been extended to the (100) interface by Saito and Ohdomari 72 . They identify a sequence of nine dangling bond jumps that result in the crystallization of three atoms. The resulting value of 1/3 for $r$ is also roughly consistent with Spaepen and Turnbull's picture for the (111) interface. However, the Arrhenius behavior of the measured SPEG rate (Fig. 9) implies that 


$$
k^{-} \ll k^{+},
$$

or $-\Delta G^{\mathrm{o}} / k T \gg 1$, which means that $r$ cannot be too small. It should be noted that, in marked contrast to the uniformity of the migration barriers postulated in Fig. 18, Saito and Ohdomari's analysis of the energetics of their nine-step bond-rearrangement process using the Keating potential indicated a significant site-to-site variability in the barrier to motion. Because the kinetics of motion in this case can be represented satisfactorily using a constant-barrier model with a suitably chosen effective barrier height ${ }^{73}$, we have chosen not to introduce the additional complication of varying barrier heights into our analysis at this time.

We approximate the normal mode frequency as

$$
\mathrm{v} \approx \mathrm{v}_{\mathrm{s}} / \lambda
$$

where $\mathrm{V}_{\mathrm{S}}$ is the speed of sound, and combine (16)-(20) to yield

$$
\left\langle\mathrm{v}_{\mathrm{db}}\right\rangle \approx \mathrm{v}_{\mathrm{s}} \exp -\left(\frac{\Delta G_{\mathrm{m}}}{\mathrm{kT}}\right)
$$

To find the steady-state probability that a bond is dangling, we balance the rates of bond rupture and of dangling bond annihilation:

$$
0=\frac{\mathrm{d}}{\mathrm{dt}} N_{\mathrm{db}}=2 k_{\mathrm{diss}} N_{\mathrm{b}}-\left(k^{+}+k^{-}\right) N_{\mathrm{db}}\left(\frac{N_{\mathrm{trap}}}{N_{\mathrm{b}}}\right)-2\left(k^{+}+k^{-}\right)\left(\frac{N_{\mathrm{db}}^{2}}{N_{\mathrm{b}}}\right),
$$

where $k_{\text {diss }}$ is the frequency of thermal bond breaking to form a pair of dangling bonds, given by

$$
k_{\mathrm{diss}} \approx v \exp -\left(\frac{\Delta G_{\mathrm{f}}+\Delta G_{\mathrm{m}}}{\mathrm{kT}}\right)
$$

$\Delta G_{\mathrm{f}}$ is the standard free energy to form a pair of dangling bonds, or the "bond energy". $N_{\text {trap }}$ in (22) is the number of "traps" per unit area of interface, into which a dangling bond may fall and either annihilate or merely remain immobilized. The nature of the traps is at present unclear; they may be immobile dangling bonds, or local bonding configurations that for energetic reasons prevent a mobile dangling bond from advancing. As will be seen, however, unimolecular annihilation kinetics at the "traps" are essential to the success of the model. Unimolecular annihilation kinetics would also result if the traps were mobile dangling bonds so long as pairs of dangling bonds, formed from the rupture of a single bond, remained within a short distance of each other during the bond rearrangement process, eventually annihilating each other.

The first term on the right-hand side of (22) represents dangling bond generation by bond rupture, the second term represents unimolecular annihilation at traps, and the final term represents bimolecular annihilation at other independently-generated mobile dangling bonds. We assume that the third term is negligible compared to the second term, in which case

$$
n_{\mathrm{r}}=N_{\mathrm{b}} / N_{\text {trap }}
$$

This assumption, combined with (15), (17), (19), and (22) - (24), results in a simple expression for the probability of finding a dangling bond at a particular site: 


$$
P_{\mathrm{db}}=2 n_{\mathrm{r}} \exp -\left(\frac{\Delta G_{\mathrm{f}}}{k T}\right) .
$$

A consequence of the above assumptions is that $n_{\mathrm{r}}$, the net number of hops made by a thermally generated dangling bond before it is trapped, is independent of temperature and pressure. (25):

The resultant expression for the growth velocity is obtained by combining (14), (21), and

$$
\mathrm{v}=2 \sin (\theta) \mathrm{v}_{\mathrm{s}} n_{\mathrm{r}} \exp \left(\frac{\Delta S_{\mathrm{f}}+\Delta S_{\mathrm{m}}}{k}\right) \exp -\left(\frac{\Delta H_{\mathrm{f}}+\Delta H_{\mathrm{m}}}{k T}\right)
$$

where $\Delta S_{\mathrm{f}}$ and $\Delta H_{\mathrm{f}}$ are the standard entropy and enthalpy of formation of a pair of dangling bonds, and $\Delta S_{\mathrm{m}}$ and $\Delta H_{\mathrm{m}}$ are the entropy and enthalpy of motion of a dangling bond at the interface. This is similar to Spaepen and Turnbull's expression ${ }^{20}$, but ours is somewhat more general. Their analysis was based on a negligible barrier to motion relative to the bond energy; ours is valid for arbitrary combinations of formation and motion barriers. Furthermore, they did not deal explicitly with the annihilation kinetics of the dangling bonds. We have shown that equation (26) is obtained if and only if the annihilation kinetics are unimolecular.

\subsection{Comparison of model to data}

In this section, we compare the model to the experimental results, deducing parameter values by fitting to the data, and we examine the parameter values for reasonableness.

Prefactor: The ambient-pressure SPEG rates of (100) Si and Ge are of the Arrhenius form

$$
\mathrm{v}(T)=\mathrm{v}_{\mathrm{o}} \exp -\left(\frac{\Delta E^{*}}{k T}\right)
$$

with prefactors of $\mathrm{v}_{\mathrm{O}}=3.1 \times 10^{6}$ and $1.2 \times 10^{7} \mathrm{~m} / \mathrm{s}$ in $\mathrm{Si}$ and Ge, respectively. The best-fit parameters to eq. (27) are taken from this study for Ge, and from Olson and Roth ${ }^{3}$ for Si. There is relatively little experimental uncertainty (about a factor of 2) in the value of the Si prefactor but an uncertainty of a factor of $\sim 50$ in the Ge value due to the small temperature range over which measurements have been made. Within the context of the model, the prefactor is

$$
\mathrm{v}_{\mathrm{o}}=2 \sin (\theta) \mathrm{v}_{\mathrm{S}} n_{\mathrm{r}} \exp \left(\frac{\Delta S_{\mathrm{f}}+\Delta S_{\mathrm{m}}}{k}\right),
$$

with $\theta=55^{\circ}$ for the (100) interface. In the model $\mathrm{v}_{\mathrm{S}}$ is actually the speed of sound at the interface which, of course, has not been measured. The measured values in the bulk phases 74 are $8.3 \mathrm{~km} / \mathrm{s}$ in c-Si, $6.3 \mathrm{~km} / \mathrm{s}$ in a-Si, $4.9 \mathrm{~km} / \mathrm{s}$ in c-Ge, and $4.0 \mathrm{~km} / \mathrm{s}$ in a-Ge; we use the speed of sound in the amorphous phases to compare eq. (26) to the data. We also assume that on average, $r$ $=1 / 3$ in accordance with Saito and Ohdomari's sequence ${ }^{72}$, i.e., three dangling bond migration events are needed to transfer one atom from the amorphous phase to the crystal. These assumptions result in 


$$
N_{\mathrm{r}} \exp \left(\frac{\Delta S_{\mathrm{f}}+\Delta S_{\mathrm{m}}}{k}\right)=\left\{\begin{array}{c}
200 \text { in } \mathrm{Si} \\
1200 \text { in Ge }
\end{array}\right.
$$

The entropy factors are difficult to evaluate. If we assume a lower bound of zero and an upper bound corresponding to the entropy of fusion, then $5 \leq N_{\mathrm{r}} \leq 200$ in $\mathrm{Si}$, and $30 \leq N_{\mathrm{r}} \leq 1200$ in Ge. This uncertainty in Ge should be combined with the additional $50 \times$ uncertainty in the measured prefactor. The result is consistent with Spaepen and Turnbull's idea that a single bond-breaking event can result in multiple crystallization events. Yet these $N_{\mathrm{r}}$ values are much more plausible than $10^{8}$, which was necessary to reconcile bulk crystal defect mechanisms to the data (Section 4.4; Table VI).

Orientation dependence: The model predicts a proportionality between $\mathrm{V}$ and $\sin (\theta)$, in agreement with the well-known results of Csepregi et al. ${ }^{1}$. Spaepen and Turnbull noted that in order to fit the data of Csepregi et al. with $\sin (\theta)$ dependence, there is a discrepancy of about a factor of 2 between the prefactors needed for an interface tilting off the (111) toward the (110) and for an interface tilting off the (111) toward the (001) direction. They explained this observation by noting the different atomic structure of $\left[\begin{array}{lll}1 & -1 & 0\end{array}\right]$ ledges in these two cases.

Activation energy: Within the context of the model, the difference between the measured activation energy for SPEG and the bond energy is the migration energy. The measured activation energies are $\Delta E_{\mathrm{SPEG}}{ }^{*}=2.68 \pm 0.05 \mathrm{eV}$ in $\mathrm{Si}$, and $\Delta E_{\mathrm{SPEG}}{ }^{*}=2.17 \pm 0.2 \mathrm{eV}$ in Ge. The Si-Si and Ge-Ge bond energies, respectively, are 1.83 and $1.63 \mathrm{eV}$, according to Pauling 75 . However, a bond-counting argument based on the cohesive energy of the crystal yields higher values of 2.315 and $1.925 \mathrm{eV}$, respectively, and we will use these latter vaules in our analysis of the SPEG process. Bond energies in the bulk of the amorphous phase are then obtained by subtracting the enthalpy of crystallization per bond, yielding $\Delta E_{\mathrm{f}}^{\mathrm{Si}}=2.246 \mathrm{eV}$ and $\Delta E_{\mathrm{f}}^{\mathrm{Ge}}=1.865 \mathrm{eV}$. We have neglected that in the network models of Spaepen and of Saito and Ohdomari, bonds at the c/a interface are slightly weaker than the values in the amorphous phase due to excess bond strain energy at the interface ${ }^{76}$. For our kinetic model to be consistent with the data, then, $\Delta E_{\mathrm{m}}=0.43$ $\mathrm{eV}$ for $\mathrm{Si}$ and $0.3 \mathrm{eV}$, with a very large uncertainty (at least $\pm 0.2 \mathrm{eV}$ ), for Ge. The value for $\mathrm{Si}$ is comparable to apparent activation energies of $0.2-0.33 \mathrm{eV}$ for ion-beam-enhanced SPEG in $\mathrm{Si}^{7,8,11}$. We are unaware of comparable measurements in $\mathrm{Ge}$.

That $\Delta E_{\mathrm{SPEG}} \stackrel{*}{*}=\Delta E_{\mathrm{m}}+\Delta E_{\mathrm{f}}$ is not necessarily the case for all models. For example, if we had assumed no traps, dropping the second term on the r.h.s. of eq. (22) instead of the third term, the resulting bimolecular annihilation process would result in $\Delta E_{\mathrm{SPEG}}{ }^{*}=\Delta E_{\mathrm{m}}+\frac{1}{2} \Delta E_{\mathrm{f}}$, leading to a migration energy (1.56 eV and $1.27 \mathrm{eV}$ in $\mathrm{Si}$ and $\mathrm{Ge}$, respectively) that we feel to be less plausible in light of the apparent activation energy for ion-beam-enhanced SPEG quoted above.

Activation volume: According to the model $\Delta V_{\mathrm{m}}$, the volume of motion of an individual dangling bond, is large and negative. A quantitative theoretical value is not presently available, but it could be within reach with the advent of modern computational techniques. According to the kinetic analysis,

$$
\Delta V_{\mathrm{SPEG}}^{*}=\Delta V_{\mathrm{m}}+\Delta V_{\mathrm{f}}
$$


where $\Delta V_{\mathrm{f}}$ is the formation volume of a pair of dangling bonds. Estimates of $\Delta V_{\mathrm{f}}$ and measurements of $\Delta V_{\mathrm{SPEG}}^{*}$ permit the assignment of approximate numerical limits to the volume of motion. For dangling bonds, $\Delta V_{\mathrm{f}}$ is clearly nonnegative but is probably quite small. Since a vacancy might be thought of as two pair of dangling bonds, an upper limit might be half the volume of formation of a vacancy (we use for the vacancy formation volume in both $\mathrm{Si}$ and Ge the theoretical value of $0.75 \Omega$ calculated 66 for $\mathrm{Si}$ ). It seems, however, that relaxation around an isolated dangling bond should be more efficient than around a vacancy, so the true value of $\Delta V_{\mathrm{f}}$ should lie closer to the lower than to the upper limit. From (30), $-0.3 \geq \Delta V_{\mathrm{m}} / \Omega_{\mathrm{Si}} \geq-0.7$ for $\mathrm{Si}$, and $-0.45 \geq \Delta V_{\mathrm{m}} / \Omega_{\mathrm{Ge}} \geq-0.8$ for Ge, where we expect the less negative values to be better approximations than the more negative values.

In principle, a structural model should predict the entire strain tensors for dangling bond formation and for dangling bond motion, the traces of which are the volumes considered above. Any successful model should be able to match all elements of the measured activation strain tensor. This has not yet been attempted, as Spaepen's ball-and-stick model is for the (111) interface and our measurements are for the (100) interface. An important future test of the model would be the construction of a (100) interface and the evaluation of the activation strain tensor. This might best be done using modern numerical computational techniques.

Free energy catastrophe: The negative volume of motion deduced above from the kinetic analysis means that the application of pressure reduces the barrier to motion. As a consequence, at high enough pressures the barrier will vanish. Dangling bonds may migrate athermally through the network or, more likely, something wildly nonlinear and presently unpredictable might be observed. An estimate of the critical pressure, $P_{\text {crit }}$, at which this "catastrophe" will occur is obtained from the energy of migration and the volume of migration inferred above. Neglecting the entropy of migration, the Gibbs free energy of migration will vanish approximately when

$$
\Delta H_{\mathrm{m}}=\Delta E_{\mathrm{m}}+P_{\text {crit }} \Delta V_{\mathrm{m}} \rightarrow 0 \text {. }
$$

If the volume of formation is zero (its lower limiting value assigned above), $\Delta V_{\mathrm{m}}=\Delta V^{*}$ and we obtain an upper limit on $P_{\text {crit }}$ of approximately $12 \mathrm{GPa}$ in $\mathrm{Si}$ and $6 \mathrm{GPa}$ in $\mathrm{Ge}$. If the formation volume is equal to its upper limiting value assigned above, $\Delta V_{\mathrm{m}}$ is even more negative and we reach our catastrophe at lower pressures - approximately $5 \mathrm{GPa}$ in $\mathrm{Si}$ and $3 \mathrm{GPa}$ in $\mathrm{Ge}$.

Shimomura et al. ${ }^{39}$ have observed at room temperature a structural transition to a metallic phase at $10 \mathrm{GPa}$ in a-Si and at $6 \mathrm{GPa}$ in a-Ge, as shown in Fig. 19. They mention a "hysteresis," indicating that this may be a kinetic, rather than a thermodynamic, transition point. The good agreement with values of $P_{\text {crit }}$ calculated above may be a coincidence, or it may be a manifestation of the "free energy catastrophe." It should also be noted that a similar analysis 77 can be applied to Fratello et al.'s measurements 78 of pressure-enhanced growth of quartz from fused silica, to which the dangling bond model was first successfully applied ${ }^{70}$. The distribution among formation and migration terms is not known, but the application of eq. (31) to $\Delta E^{*}$ and $\Delta V^{*}$ yields a catastrophe pressure of $12.5 \mathrm{GPa}$, by which point either the barrier to formation or the barrier to motion should have vanished. This interpretation is supported by the observation at room temperature of pressure-induced structural changes generally not expected to be observable below about $1000^{\circ} \mathrm{C}$ - reversible coordination changes in fused silica near $20 \mathrm{GPa}^{79}$, the amorphization of quartz at 20-30 $\mathrm{GPa}^{80}$, and the crystallization of amorphous silica at 60-70 
$\mathrm{GPa}^{81}$.

\subsection{Doping Dependence}

Although this model does not address the dopant-induced enhancement of the SPEG rate directly, we note that the population or mobility of charged dangling bonds at the interface could respond to the doping level through a variety of schemes discussed in the literature $6,13,15,82$. The details are still a matter of debate. We summarize, critique, or generalize a number of models below, keeping in mind that the assumptions about the structural identities of the defects and those about the doping-induced enhancements of their populations or mobilities are in many cases independent of each other and need not stand or fall together. The models that appear most successful at this time are those invoking an enhancement in the concentration of charged defects with doping. Currently at issue is whether the response of the concentration of charged defects to the doping level can be computed using the electronic structure of the crystal or of the amorphous phase.

There have been numerous experimental studies of the doping-induced enhancement of the SPEG rate and the related compensation effect, in which equal amounts of donors and acceptors offset each other resulting in a growth rate characteristic of undoped material. The Walser group 42,43 has performed the most thorough experimental investigation to date. They found experimentally that for B, P, or As (but not Al) in (100) Si the concentration-dependence of the SPEG rate can under some circumstances be expressed as

$$
\mathrm{v}\left(N_{D}, T\right)=\mathrm{v}_{\mathrm{i}}(T)\left(1+\frac{N_{D}}{N_{\mathrm{norm}(T)}}\right)
$$

where $N_{D}$ is the dopant concentration at the interface, $\mathrm{v}_{\mathrm{i}}(T)$ is the growth velocity in intrinsic $\mathrm{Si}$, and the proportionality factor $N_{\text {norm }}(T)$ has Arrhenius form,

$$
N_{\text {norm }}(T)=N_{o} \exp -\left(\frac{Q_{N}}{k T}\right)
$$

with a temperature-dependence, $Q_{N}$, of only $\sim 0.3 \mathrm{eV}$ and a prefactor, $N_{o}$, of approximately $3 \times$ $10^{21} / \mathrm{cm}^{3}$. The uncertainty in $Q_{N}$ is about $0.04 \mathrm{eV}$ and that in $N_{o}$ is about a factor of two, although the uncertainty in $N_{\text {norm }}(T)$ at SPEG temperatures is quite a bit smaller ${ }^{83}$. This relation seems to hold when the interface position is between the peak of the implant and the free surface and when the concentration is between a few $\times 10^{18} / \mathrm{cm}^{3}$ and a few $\times 10^{19} / \mathrm{cm}^{3}$, depending on dopant species. These doping levels make the carrier concentration in the crystal extrinsic (i.e., concentration of majority carriers = concentration of dopants) in the temperature range over which the measurements were made.

Charged vacancy model. Suni et al. ${ }^{6}$ assumed that vacancies in the bulk of the crystal control the SPEG rate. This is a Fermi-level shifting (FLS) model, in that the concentration of charged vacancies is determined by the proximity of the charged vacancy level to the Fermi level. According to this model,

$$
\mathrm{v}(c, T)=\mathrm{v}_{\mathrm{i}}(T) \exp +\left(\frac{E_{\mathrm{F}}-E_{\mathrm{i}}}{k T}\right)
$$

where $\mathrm{V}_{\mathrm{i}}(T)$ is the growth rate for intrinsic material, $E_{\mathrm{F}}$ is the Fermi level of doped material, and 
$E_{\mathrm{i}}$ is the Fermi level in intrinsic material. The association of the SPEG rate with crystal vacancies has been ruled out in our discussion above (Section IV), but here we examine the electronic aspects of the model. The assumption that doping will increase the concentration of charged defects without altering the concentration of neutral defects is in accordance with the law of mass action. However, to derive eq. (34), the implicit assumption must also be made that neutral defects, no matter how plentiful, do not contribute in any significant manner to the SPEG process. Otherwise, an additional term arising from neutral defects must be added to the r.h.s. of eq. (34). FLS models resulting in equations of the form of (34) have been criticized by the Walser group ${ }^{42}$ on the basis that they predict the wrong concentration-dependence. Indeed, we find this criticism to be valid. Writing the r.h.s. of eq. (34) as a function of $N_{D}$ to compare with eq. (32), a $\mathrm{v}\left(N_{D}\right)$ curve is obtained that, when extrapolated linearly from the region covered by the experiments, passes directly through the origin.

Electric field models. Mosley and co-workers $15,84,85$ and Licoppe and Nissim 17 assumed that the diffusion of charged defects (dangling bonds in the case of Mosley et al.) from the bulk of the amorphous phase to the c/a interface controlled the SPEG rate. Because the Fermi level in the amorphous phase is pinned near mid-gap, doping causes a band-bending effect, creating an electric field that attracts charged defects to the interface. The barrier to dangling bond motion is then assumed to be reduced by an electrostatic potential difference, and this is assigned to the reduction in the apparent activation energy for growth. We believe that these models, if developed properly, cannot predict a large enough doping effect to match the data. The field-induced reduction in the barrier to motion of charged defects should be approximately half the product of the electric field and the jump distance - a very small number. In order to predict a significant effect, Mosley et al. assume that the barrier to each jump of the dangling bond through the amorphous phase is reduced by the entire potential difference between the bulk of the two phases ${ }^{15}$. Licoppe and Nissim modify the potential change per jump with an effective jump length that accounts for the lack of a barrier change for neutral defects and allows for a defect jump distance of arbitrary size. To obtain an effect large enough to match the experimental results, the defect jump length must be huge $(20 \AA)$ if there are no neutral defects, and even larger if there are neutral defects. With reasonable numbers for the defect jump distance, the effect of doping on the apparent activation energy is at best twenty times too small to account for the data; it is smaller still if neutral defects contribute at all to the process. Furthermore, we have shown above that the only significant barrier to motion is for motion along the interface. Such a barrier would not be affected by an electric field directed perpendicular to the interface. Despite these difficulties, the model of Mosley et al. is the only one that we know of to address the experimental observations of "asymmetry": that $v$ is not a unique function of concentration, but rather depends upon whether the interface is deeper than or shallower than the peak of the implant profile.

Fractional ionization model. Walser and co-workers $42,43,82,86$ attribute the dopingenhancement term in eq. (32) to the presence of charged defects moving with an activation energy that is $Q_{\mathrm{N}}$ lower than the neutral defects that dominate the intrinsic behavior. The concentration of charged defects is enhanced by doping; the implicit assumption is made that the concentration of neutral defects is independent (at least of small amounts) of doping. The model does not require that the structural nature of the defects be specified, so long as their populations are determined by the band structure of the amorphous phase. In recent versions of the model, the defect is assumed to be a dangling bond; the model need not predict whether the operative dangling bonds are in the bulk of the amorphous phase or at the c/a interface. In one publication 82 it is mistakenly ${ }^{83}$ stated that eq. (32) follows from an analysis of the statistics of charged dangling bond populations based on the assumption that the dangling bonds, band tail states, and impurity states in the amorphous phase are in a state of metastable equilibrium governed by the laws of mass action and charge neutrality. However, the assumption of mass action leads to the final term in (32) being 
proportional to $N_{D}^{1 / 2}$, because mass action implies the doping efficiency 87 in a-Si varying as $N_{D}{ }^{-1 / 2}$. The authors recognize this $N_{D}{ }^{1 / 2}$ behavior elsewhere 86 . Clearly, the minimal set of assumptions to obtain eq. (32) is that the concentration of neutral defects be independent of dopant concentration and that the concentration of charged defects be proportional to the net dopant concentration. The latter can be derived from charge neutrality in the amorphous phase and the assumption of a concentration-independent doping efficiency. Walser et al. derive eq. (32) from the assumptions of (i) charge neutrality and (ii) that the fractional ionization of dopant atoms in a-Si is independent of concentration, which implies that the law of mass action is inoperative in this case. Assumption (ii), which is not unique to this model of $\mathrm{SPEG}^{88}$, has no a priori justification that we know of; nor have we been able to derive it from other principles. Furthermore, with mass action out the window, the source of the compensation effect is not obvious. Nevertheless, the parameters extracted to fit the data to eq. (32) are quite reasonable, as discussed below. Within the context of this model, the temperature-dependence shown in eq. (33) could arise from a temperature-dependence of the ratio of the fractional ionization to the concentration of neutral dangling bonds, or from a different migration enthalpy for charged and neutral dangling bonds. The authors assume different migration enthalpies, but identical migration prefactors, for charged and neutral dangling bonds; they also assume the ratio of the fractional ionization to the concentration of neutral dangling bonds to be temperature-independent. These assumptions could be relaxed in the future if the circumstances so warranted.

Charged kink-site model. The structural aspect of the Williams-Elliman kink mode1 13,89 seems to be a special case of the Spaepen-Turnbull dangling bond model, where the dangling bonds reside at kink sites on ledges separating $\{111\}$ terraces. All sites on a flat (100) c/a interface are already at such ledges, and (based on a criterion of counting the number of bonds a crystallizing atom would immediately make to neighbors in the crystal) kinks on those ledges do not offer preferred sites for attachment of atoms in the crystalline configuration. Furthermore, since atoms in the crystal and the amorphous phase are expected to be for the most part fully coordinated, the only way that a kink can move is by a bond rearrangement process. This would almost certainly be brought about by the breaking of bonds across the interface to create pairs of dangling bonds, the rearrangement of the network using the dangling bonds, and the recombining of the dangling bonds. One might then interpret the kink site model as the special case of the dangling bond model in which $\mathrm{n}_{\mathrm{r}}$, the number of jumps of a dangling bond before annihilation, takes its minimum possible value.

The main emphasis of the kink-site model is its electronic aspect. The increase in $\mathrm{V}$ due to doping is attributed to the shift of the Fermi level toward a defect state in the band gap of the crystal, creating more charged defects while not affecting the concentration of neutral defects in accordance with the laws of mass action. This defect, called a kink site by the authors, could easily be that of a dangling bond at the interface; we will assume this below. This model is also a Fermi-level shifting model, but the inclusion of neutral defects in the process keeps it from leading to eq. (34), which does not fit the data. Williams and Elliman made a number of simplifying assumptions that place it in conflict with the most recent experimental results. However these assumptions are unnecessarily restrictive, and we find that if they are relaxed the model is more successful at fitting the data. In Appendix A we repeat their analysis, relaxing their assumptions (i) that the mobilities of charged and uncharged dangling bonds are identical, (ii) that the temperature-dependence of the band gap can be neglected, and (iii) the heavy-doping limit that the concentration of charged defects exceeds the concentration of neutrals. We also explicitly deal with the temperature-dependence of the effective density of states in the conduction band edge. We treat only the case of doping with donors, the extension to doping with acceptors being straightforward. We show that this approach matches the experimental results described by eqs. (32) and (33).

The interpretation of the parameters in eq. (33) using the generalized FLS and fractional- 
ionization (FI) models are quite different. In the FI model $N_{o}=\left[\mathrm{D}^{\mathrm{o}}\right] / \alpha$, where $\left[\mathrm{D}^{\mathrm{o}}\right]$ is the concentration of neutral dangling bonds and $\alpha$ is the fraction of dopants that are ionized in the amorphous phase; and $Q_{\mathrm{N}}=\Delta E_{\mathrm{m}}^{\mathrm{O}}-\Delta E_{\mathrm{m}}^{-}$is the difference in the energies of motion of neutral and charged dangling bonds. This identification of parameters is subject to the assumptions discussed above. If $\left[\mathrm{D}^{\mathrm{O}}\right]$ is taken to be the density of unpaired electrons in the bulk of a-Si, measured to be $\approx 10^{19} / \mathrm{cm}^{3}$ with electron spin resonance ${ }^{50}$, then to fit the data $\alpha$ must be about $0.3-0.5 \%$. These values for $\alpha$ are in excellent agreement with the values of $0.2-1 \%$ estimated from photoconductivity measurements 88 . In the generalized FLS model,

$$
N_{\text {norm }}(T)=\frac{N_{C} A^{\mathrm{o}}}{g A^{-}} \exp -\left(\frac{E_{C}-E^{-}+\Delta E_{\mathrm{m}}^{-}-\Delta E_{\mathrm{m}}^{\mathrm{o}}}{k T}\right),
$$

where $E_{\mathrm{C}}$ is the conduction band edge in the crystal, $E^{-}$is the defect level in the band gap and $g$ is its degeneracy, $N_{\mathrm{C}}$ is the effective density of states in the conduction band, and $\Delta E_{\mathrm{m}}^{-}$and $\Delta E_{\mathrm{m}}^{\mathrm{o}}$ are migration energies and $A^{\circ}$ and $A^{-}$are prefactors for the mobilities of neutral and negative dangling bonds, respectively. While this expression appears to have Arrhenius form, caution must be exercised due to the temperature-dependence of $N_{C}$ and of the band gap. As discussed in Appendix A, using the usual $T^{3 / 2}$ dependence to $N_{C}$ and assuming identical mobilities for neutral and charged dangling bonds and reasonable guesses about the temperature dependence of $E_{C}-E^{-}$, the experimental value for $N_{O}$ in (33) can be matched to about a factor of two, with a theoretical uncertainty of perhaps an order of magnitude. If the mobilities are allowed to differ, any value of $N_{o}$ can be matched exactly but the parameters in (35) cannot be uniquely determined solely from a fit to the data. The problems with this model are that it postulates defect levels that have not yet been found, and that the quantitative predictions of the model are sensitive to the postulated temperature-dependence of the energy level of the defect, as discussed in Appendix A. Eq. (35) does not predict a strictly Arrhenius form for $N_{\text {norm }}(T)$; hence accurate enough data for the temperature-dependence of $v$ could in principle be used to distinguish between the FI and generalized FLS models. Additionally, attempts to fit $N_{o}$ from experiments in doped Ge might favor one model over the other, but only if one of the large number of fitting parameters were forced outside its plausible range.

In summary, the electric field models predict too small an effect when developed properly; this seems to be an insurmountable difficulty of these models. The viable models for the doping effect seem to be the generalized Fermi-level-shifting model and the fractional-ionization model, in that they both can be made to reproduce eqs. (32) and (33). They are based on entirely different assumptions. The FLS model assumes that the population of charged defects responds to the density of states of the crystal; the FI model assumes that it responds to the density of states of the amorphous phase. The main problem of the FLS model is that it depends on a defect level that is difficult to design an independent experiment to find; also its quantitative predictions are sensitive to the temperature-dependence of the defect level. The main problem with the FI model is the compensation effect in the absence of mass action. Additional challenges for both models are a prediction of the behavior at concentrations high enough that eq. (32) breaks down, and an explanation of the growth behavior asymmetry between the shallow and deep sides of implanted profiles.

\subsection{Ion-beam enhanced SPEG}


Ion irradiation alters the SPEG rate 90 . At low ion fluxes and high temperatures, the rate is enhanced by ion beams; at high ion fluxes and low enough temperatures, SPEG reverses direction and amorphization occurs by the motion of a sharp interface. While our work directly addresses thermal SPEG only, here we discuss how the ion-beam phenomena and thermal SPEG are related. Jackson ${ }^{91}$ models the process as the immediate production of a certain amount of amorphous material during an ion cascade, followed by enhanced crystallization mediated by a radiationenhanced concentration of point defects which undergo bimolecular annihilation. The difference between the rates of amorphization and of crystallization determines the direction and velocity of interface motion. Jackson's model accounts well for the linear relationship between interface motion and reciprocal temperature, for the Arrhenius behavior of the flux at which no interface motion occurs, and for the temperature-independence of the crossover frequency observed in beam-pulsing experiments. Priolo et al. ${ }^{92}$ assumed the defects in Jackson's kinetic analysis to be kink sites and combined it with a Fermi-level shifting model for doping dependence, accounting for the doping and partial compensation effect observed during ion irradiation, and for the limited orientation-dependence measurements that have been made to date.

The structural nature of the defects in Jackson's model need not be specified, but he speculates that they may be dangling bonds. Bimolecular annihilation kinetics are essential to the model in order to obtain any temperature dependence of the ion flux at which the interface does not move. Within the context of the Jackson model, this temperature dependence $(1.2 \mathrm{eV})$ is that of defect motion, $\Delta E_{\mathrm{m}}$. A consequence of bimolecular annihilation kinetics is that the measured activation energy for thermal SPEG $(2.7 \mathrm{eV})$ is the sum of the energy of motion of a single defect and half the energy of formation of a pair of defects (section 4.9). This is the reason for Jackson's observation that if the same defects responsible for thermal SPEG are to be responsible for beamenhanced SPEG, his model requires a formation energy of $3 \mathrm{eV}$ per defect pair in $\mathrm{Si}$. This value seems far too large (section 4.9) for a bond energy in Si. As in eq. (22), a transition is possible, from bimolecular annihilation kinetics occurring at the high dangling bond densities resulting from collision cascades, to unimolecular annihilation kinetics in thermal SPEG when the ion beam is reduced and dangling bonds are far less numerous. In this case, Jackson's identification of $1.2 \mathrm{eV}$ as the migration energy still stands, and the bond energy would have to be $1.5 \mathrm{eV}$. This value seems somewhat low (section 4.9), although in a covalent network with significant bond-angle distortion, structural relaxation around the newly-formed dangling bonds might lower their formation energy to this value. If we assume the heat of crystallization to be entirely due to bondangle distortion, then the complete relaxation of 14 average bond angles would account for the difference between Pauling's $1.83 \mathrm{eV}$ bond energy and the value of $1.5 \mathrm{eV}$ inferred here. If the migration energy were lower by $0.1 \mathrm{eV}$, which seems to be within the realm of experimental uncertainty, the relaxation of only 10 average bond angles would be necessary. We expect the immediate and complete relaxation of three bond angles at each of the two atoms hosting a newlyformed dangling bond. Furthermore, the bonds most likely to break may be associated with above-average bond-angle distortion. Preliminary results of numerical calculations ${ }^{93}$ on the Polk continuous random network model ${ }^{94}$ of this effect, using the second-order Keating potential ${ }^{95}$ with parameters determined by the elastic constants of c-Si, yield relaxation energies of $0.2-0.7$ $\mathrm{eV}$.

An alternative hypothesis is that the Spaepen-Turnbull dangling bond mechanism might occur with bimolecular annihilation kinetics even in thermal SPEG; in this case, however, either the energy of formation or of motion must be significantly greater than the values derived in section 4.9. The main consequences would be that either the energy of migration is higher than the $1.2 \mathrm{eV}$ inferred from the Jackson model's interpretation of the ion beam data (and the "free energy catastrophe" moves to irrelevantly high pressures) or the bond energy is much greater than the value inferred from the cohesive energy. We feel that the most plausible scenario is the transition from bimolecular to unimolecular annihilation kinetics. 
It is also possible that different defects are responsible for thermal SPEG and beamenhanced SPEG. Experiments comparing the effects of channeled and random ion beams on the SPEG rate ${ }^{96}$ have been interpreted to imply a contribution from bulk crystal point defects. However, any potential role of bulk point defects from either phase in beam-enhanced SPEG has been limited by other similar experiments 8,97 and by the lack of an observed amorphous-layer thickness dependence to the rate during ion bombardment ${ }^{8}$. Our work on thermal SPEG implies that ion beam-enhanced SPEG may involve, for example, bulk point defects of any type impinging on the interface and converting to interfacial dangling bonds. But when the ion beam is turned off, the rate of interface motion cannot be limited by the arrival rate of these suddenly-less-numerous defects. Ion-beam induced bulk point-defect mechanisms, operating in parallel with thermal generation of dangling bonds at the interface, are also possible. For any of these scenarios to be given much credence, the transition from beam-enhanced SPEG to thermal SPEG as the ion flux is lowered must be addressed quantitatively.

Priolo et al.'s synthesis of the Jackson and Williams-Elliman models avoids some of these difficulties because it invokes a beam-enhanced population of interfacial defects. We see nothing in their model that prevents it from applying to dangling bonds at the interface. However, the authors use a restricted version of the FLS model; consequently, to fit the data they must assume a fraction of electrically active dopants of $2 \%$ in the crystal. This problem could be eliminated by employing the generalized FLS model from Appendix A.

\section{Conclusions}

We summarize our conclusions for thermal SPEG in Table I. Bulk crystal point-defect mechanisms are untenable because of the magnitude and sign of the activation volume for SPEG and because of the low bulk crystal diffusivity. Bulk amorphous point-defect mechanisms are untenable because uniaxial compression reduces $\mathrm{V}$ while hydrostatic pressure enhances $\mathrm{V}$, and because the relaxation behavior for SPEG is different than that for diffusion. Our results are consistent with any mechanism involving defect formation or motion at the interface. A kinetic analysis of the Spaepen-Turnbull dangling bond mechanism, postulating thermal generation of dangling bonds at the interface and unimolecular annihilation kinetics at "traps", shows it to be a highly plausible model for the growth process. The nature of the "traps" is at present unclear. The Williams-Elliman kink model seems to be a special case of the dangling bond model, where the dangling bonds reside at kink sites and the number of jumps of a dangling bond before annihilation takes its minimum possible value. Within the context of our kinetic analysis, the number of atoms crystallized per dangling bond formed is between 5 and $200 \mathrm{in} \mathrm{Si}$, and 30 and 1200 in $\mathrm{Ge}$, which makes the limiting case of the kink model seem somewhat low. For both Si and Ge the model gives plausible semi-quantitative interpretations to the prefactor, orientation dependence, activation energy, and activation volume of SPEG, and to the maximum pressure attainable before a "free energy catastrophe" eliminates the barrier to motion of dangling bonds.

The doping-dependence might be explained by the density of states of the amorphous phase through the fractional-ionization model of Walser and co-workers, or by the density of states of the crystal through a generalized Williams-Elliman Fermi-level shifting model. As we have shown that the relevant defects reside at the c/a interface, it might now be appropriate to call for a third type of doping model based on the density of states in the interface.

These conclusions are valid for thermal SPEG only. Ion irradiation may alter the populations of dangling bonds in a manner described by the Jackson model, with a transition from bimolecular to unimolecular annihilation kinetics as the ion beam is turned off. Our work on thermal SPEG 
implies that ion beam-enhanced SPEG may involve, for example, bulk point defects of any type impinging on the interface and converting to interfacial dangling bonds. But when the ion beam is turned off, the rate of interface motion cannot be limited by the arrival rate of these suddenly-lessnumerous defects. It may also involve alternative point-defect mechanisms operating in parallel with thermal generation of dangling bonds at the interface.

We are grateful to Byungwoo Park (IBM) for valuable assistance with the relaxation experiment, to G.J. Piermarini (NIST) for providing us with his DAC blueprints and many helpful discussions concerning the design of and use of the diamond anvil cell, and to G.L. Olson (Hughes) for valuable advice in setting up the TRR system. We also thank Dr. K. Goettel, Prof. I. F. Silvera, and J. Burnett for their help with pressure measurements and P. H. Fleming (ORNL) for her skilled assistance with sample preparation. We are grateful to F. Spaepen and D. Turnbull for helpful discussions on structural aspects, and to R.M. Walser for helpful discussions on electronic aspects, of the modeling. This research was supported initially by the Harvard Materials Research Laboratory under NSF-DMR-86-14003 and subsequently by the NSF-PYIA program matching an IBM Faculty Development Award. The portion of the research dealing with nonhydrostatic stress effects was supported by NSF-DMR-89-13268.

\section{Appendix A. Generalized Fermi-Level Shifting Model}

Assume that SPEG occurs through the motion of some neutral defect $D^{o}$ and its negatively charged counterpart $D^{-}$. The generalization to positively charged defects is straightforward and will be left out. Assume also that each charge state is equally efficient in promoting the SPEG process (i.e., equal mobilities, etc., an assumption that is relaxed later) so that the SPEG rate can be written

$$
\mathrm{v}=A\left(\left[D^{o}\right]+\left[D^{-}\right]\right)
$$

where square brackets denote concentrations and the constant $\mathrm{A}$ is independent of the defect concentrations. Assume furthermore that these interfacial defects are in thermal and electronic equilibrium, and that the latter is determined by the band structure and density of states of the crystal. The equations for electronic equilibrium and the parameter values quoted below are taken from Sze ${ }^{98}$. The concentrations of neutral and charged defects are then given by

$$
\left[D^{o}\right]=f(T, P),
$$

where $f$ is some function that is independent of the doping level, and

$$
\frac{\left[D^{-}\right]}{\left[D^{o}\right]}=g \exp \left(\frac{E_{F}-E^{-}}{k T}\right)
$$

where $E_{F}$ is the Fermi energy in the crystal, $E^{-}$and $g$ are the energy and degeneracy of the $D^{-}$ electronic state. The SPEG velocity can then be rewritten 


$$
\begin{aligned}
& \mathrm{v}=A\left[D^{o}\right]\left(1+\frac{\left[D^{-}\right]}{\left[D^{o}\right]}\right) \\
& =A\left[D^{o}\right]\left(1+g \exp \left(\frac{E_{F}-E^{-}}{k T}\right)\right)
\end{aligned}
$$

The ratio of SPEG rates in doped and intrinsic materials is given by

$$
\begin{gathered}
\frac{\mathrm{v}}{\mathrm{v}_{i}}=\frac{1+\left.\frac{\left[D^{-}\right]}{\left[D^{o}\right]}\right|_{\text {doped }}}{1+\left.\frac{\left[D^{-}\right]}{\left[D^{o}\right]}\right|_{\text {intrinsic }}} \\
=\frac{1+g \exp \left(\frac{E_{F}-E^{-}}{k T}\right)}{1+g \exp \left(\frac{E_{F, i}-E^{-}}{k T}\right)}
\end{gathered}
$$

where $\mathrm{v}_{\mathrm{i}}$ and $\mathrm{E}_{\mathrm{F}, \mathrm{i}}$ are the SPEG rate and Fermi level in intrinsic material. $E_{F}$ is determined from an expression for charge neutrality and mass action in a non-degenerate semiconductor:

$$
N_{C} \exp \left(\frac{E_{F}-E_{C}}{k T}\right)=\frac{N_{D}}{2}+\sqrt{\left(\frac{N_{D}}{2}\right)^{2}+\left(N_{C} \exp \left(\frac{E_{F, i}-E_{C}}{k T}\right)\right)^{2}}
$$

where $N_{D}$ is the concentration of dopants (assumed to be donors), $N_{C}$ is the effective density of states in the conduction band, and $E_{C}$ is the conduction band edge. The second term in the square root is the square of the intrinsic carrier concentration. If (A.7) is used to eliminate $E_{\mathrm{F}}$ from (A.4), the following expression is obtained:

$$
\frac{\mathrm{v}}{\mathrm{v}_{i}}=\frac{1+g \exp \left(\frac{E_{C}-E^{-}}{k T}\right)\left[\frac{N_{D}}{2 N_{C}}+\sqrt{\left(\frac{N_{D}}{2 N_{C}}\right)^{2}+\left(\exp \frac{E_{F, i}-E_{C}}{k T}\right)^{2}}\right]}{1+g \exp \left(\frac{E_{F, i}-E^{-}}{k T}\right)}
$$

For any given value of $E^{-}$, there is a temperature range over which this equation can be approximated by

$$
\frac{\mathrm{v}}{\mathrm{v}_{i}}=1+g \frac{N_{D}}{N_{C}} \exp \left(\frac{E_{C}-E^{-}}{k T}\right)
$$


which has the same form as the experimental result of Walser and coworkers,

$$
\frac{\mathrm{v}\left(N_{D}, T\right)}{\mathrm{v}_{\mathrm{i}}(T)}=1+\frac{N_{D}}{N_{\text {norm }}(T)}
$$

if we make the identification

$$
N_{\text {norm }}(T)=\frac{N_{C}^{e f f}}{g} \exp \left(\frac{E^{-}-E_{C}}{k T}\right)
$$

Note that $E^{-}$is a free parameter (at least until the defect level is measured) but $g$, the degeneracy of a negative dangling bond state, should be unity. Furthermore $N_{C}$, the effective density of states in the conduction band edge of the crystal, is in principle also known. However, we treat it as a free parameter $N_{C}^{e f f}$, to force an exact fit to the data represented by (32) and (33) for the discussion below. The closer the fitted value $N_{C}^{e f f}$ takes to the actual value of $N_{C}$ in c-Si, the more confidence is generated in the FLS model.

Walser and coworkers have determined Arrhenius parameters that describe the temperature dependence of the parameter $N_{\text {norm }}$ for several different dopant species:

$$
N_{\text {norm }}(T)=N_{o} \exp -\left(\frac{Q_{N}}{k T}\right)
$$

For arsenic doping over the temperature range from 470 to $580^{\circ} \mathrm{C}, N_{O}=3 \times 10^{21} / \mathrm{cm}^{3}$ and $Q_{N}=$ $0.34 \mathrm{eV}$. It is tempting to neglect the temperature dependence of $N_{C}$ and $\left(E^{-}-E_{C}\right)$, in which case if $g$ is taken to be equal to unity then a comparison of (A.10) with (33) yields $E^{-}=E_{C}-0.34 \mathrm{eV}$ and $N_{C}^{\text {eff }}=3 \times 10^{21} / \mathrm{cm}^{3}$. This value of $N_{C}^{\text {eff }}$ is too high: in c-Si, the true value of $N_{C}$ ranges from 1.1 to $1.3 \times 10^{20} / \mathrm{cm}^{3}$ over same temperature interval. The FLS model in this form, using the true value of $N_{C}$, therefore predicts too strong a doping effect. However, one cannot neglect the $T^{3 / 2}$ temperature dependence of the effective density of states in the conduction band edge (at $500^{\circ} \mathrm{C}$, $\left.\partial \ln N_{C} / \partial(1 / k T) \approx-0.1 \mathrm{eV}\right)$. Additionally, the temperature-dependence of the band gap $E_{g}$ (at $\left.500^{\circ} \mathrm{C},(-1 / k T) \partial \ln E_{g} / \partial(1 / k T) \approx-0.3 \mathrm{eV}\right)$ implies that if $\left(E^{-}-E_{C}\right)$ is independent of $T$, then $\left(E^{-}-E_{V}\right)$, where $E_{V}$ is the valence band edge, has a significant temperature dependence. We have no a priori way to predict which band edge, if either, the defect level will "track" as $T$ is varied. If we assume that the defect level tracks the valence band edge $\left(E^{-}-E_{V}=\right.$ constant $)$ and we use the usual temperature-dependent crystalline values for $E_{C}$ and $E_{F, i}$ and a T $\mathrm{T}^{3 / 2}$ temperature dependence for $N_{C}^{\text {eff }}$, we obtain $E^{-}-E_{V}=1.03 \mathrm{eV}$ and $N_{C}^{\text {eff }} / N_{C}=0.13$. If we change only the assumption that $E^{-}-E_{V}=$ constant, replacing it with $E^{-}-E_{C}=$ constant, we obtain $E^{-}=E_{C}-0.23 \mathrm{eV}$ and $N_{C}^{\text {eff }} / N_{C}=5$. If we assume instead that the defect level remains at a constant fraction of the band gap, we obtain

$$
\frac{E^{-}-E_{V}}{E_{C}-E_{V}}=0.82
$$


and $N_{C}^{e f f} / N_{C}=2.3$. We note that for $E^{-}$determined by (A.11) or any of the previous sets of assumptions, the approximation that we used to obtain (A.9) from (A.8) is valid over the range of $T$ and $N_{D}$ covered by the experiments. Finally, if the assumption of equal mobilities for neutral and charged species is relaxed, then the FLS parameter $N_{C}^{\text {eff }}$ will be multiplied by a ratio of the mobility prefactors for charged and uncharged species. The partitioning of $Q_{N}$ will also change to include the difference in barriers for motion, as shown in eq. (35). 


\section{References}

aPresent address: Alcoa Technical Center, 7th Street Road, Route 780, Alcoa Center, PA 15069. bPresent address: Department of Materials Science and Engineering, The Ohio State University, Columbus, OH 43210.

${ }^{1}$ L. Csepregi, J.W. Mayer, and T.W. Sigmon, Phys. Lett. A 54, 157 (1975).

${ }^{2}$ L. Csepregi, R.P. Kullen, J.W. Mayer, and T.W. Sigmon, Solid State Commun. 21, 1019 (1977).

${ }^{3}$ G.L. Olson and J.A. Roth, Mater. Sci. Reports $\underline{3}, 1$ (1988).

${ }^{4}$ M.G. Grimaldi, M. Maenpaa, B.M. Paine, M.-A. Nicolet, S.S. Lau, and W.F. Tseng, J. Appl. Phys. 52, 1351 (1981).

5 E.P. Donovan, F. Spaepen, D. Turnbull, J.M. Poate, and D.C. Jacobson, J. Appl. Phys. 57, 1795 (1985).

6I. Suni, G. Goltz, M.-A. Nicolet, and S.S. Lau, Thin Solid Films 93, 171 (1982).

7J. Linnros, B. Svensson, and G. Holmden, Phys. Rev. B30, 3629 (1984).

8J.S. Williams, R.G. Elliman, W.L. Brown, and T.E. Seidel, Phys. Rev. Lett. 55, 1482 (1985).

${ }^{9}$ G. Lulli, P.G. Merli, and M.V. Antisari, MRS Symp. Proc. 100, 375 (1988).

10A. La Ferla, E. Rimini, S. Cannavo, and G. Ferla, MRS Symp. Proc. 100, 381 (1988).

11F. Priolo, A. La Ferla, and E. Rimini, J. Mat. Res. $\underline{3}$, 1212 (1988).

12F. Spaepen and D. Turnbull, AIP Conf. Proc. 50, 73 (1979).

13J.S. Williams and R.G. Elliman, Phys. Rev. Lett. 51, 1069 (1983).

14J. Narayan, J. Appl. Phys. 53, 8607 (1982).

15L.E. Mosley and M.A. Paesler, Appl. Phys. Lett. $\underline{45}, 86$ (1984).

16S.T. Pantelides, MRS Symp. Proc. $\underline{100}, 387$ (1988).

${ }^{17}$ C. Licoppe and Y.I. Nissim, J. Appl. Phys. 59, 432 (1986).

18J.W. Christian, "The Theory of Transformations in Metals and Alloys, " 2nd edition, (Pergamon Press, Oxford, 1973), Chap. 11.

${ }^{19}$ D. Turnbull, J. Phys. Chem. 66, 609 (1962).

${ }^{20}$ F. Spaepen and D. Turnbull, in Laser Annealing of Semiconductors, eds. J.M. Poate and J.W. Mayer (Academic, New York, 1982), p. 15.

${ }^{21}$ G.H. Vineyard, J. Phys. Chem. Solids $\underline{3}, 121$ (1957).

22 G.Q. Lu, E. Nygren, M.J. Aziz, D. Turnbull, and C.W. White, Appl. Phys. Lett. 54, 2583 (1989).

23 G.Q. Lu, E. Nygren, M.J. Aziz, D. Turnbull, and C.W. White, Appl. Phys. Lett. 56, 137 (1990).

${ }^{24}$ G.Q. Lu, E. Nygren, and M.J. Aziz, Mater. Res. Soc. Symp. Proc. 205 (in press, 1991).

25F. Spaepen, A.L. Greer, K.F. Kelton, and J.L. Bell, Rev. Sci. Instrum. 56, 1340 (1985).

${ }^{26}$ G.J. Piermarini, S. Block, J.D. Barnett, and R.A. Forman, J. Appl. Phys. 46, 2774 (1975).

${ }^{27}$ A. Jayaraman, Rev. Sci. Instrum. 57, 1013 (1986).

${ }^{28}$ G.Q. Lu, Ph.D. Thesis, Harvard University (1990).

${ }^{29}$ R.A. Forman, G.J. Piermarini, J.D. Barnett, and S. Block, Science 176, 284 (1972).

30M. Ross, H.K. Mao, P.M. Bell, and J.A. Xu, J. Chem. Phys. 호, 1028 (1986).

${ }^{31}$ C-S. Zha and R. Boehler, J. Chem. Phys. 85, 1034 (1986).

${ }^{32}$ S. Yamaoka, O. Shimomura, and O. Fukunaga, Proc. Jpn. Acad. 56, 103 (1980).

33C-S. Zha and R. Boehler, Phys. Rev. B31, 3199 (1985).

${ }^{34}$ J.A.H. Da Jornada, S. Block, and G.J. Piermarini, Appl. Phys. Lett. 45, 700 (1984).

35G.L. Olson, S.A. Kokorowski, R.A. McFarlane, and L.D. Hess, Appl. Phys. Lett. 37, 1019 
(1980).

36G.A.N. Connell, R.J. Temkin, and W. Paul, Advances in Physics 22, 643 (1973).

${ }^{37}$ E.F. Kennedy, L. Csepregi, J.W. Mayer, and T.W. Sigmon, J. Appl. Phys. 48, 4241 (1977).

38J.A. Roth, G.L. Olson, D.C. Jacobson, J.M. Poate, and C. Kirschbaum, Mater. Res. Soc. Symp. Proc. 205 (in press, 1991).

${ }^{39}$ O. Shimomura, S. Minomura, N. Sakai, K. Asaumi, K. Tamura, J. Fukushima, and H. Endo, Philos. Mag. 29, 547 (1974).

${ }^{40}$ E. Nygren, M.J. Aziz, D. Turnbull, J.M. Poate, D.C. Jacobson, and R. Hull, Appl. Phys. Lett. 47, 232 (1985).

${ }^{41}$ M.J. Aziz, P. Sabin, and G.Q. Lu, Phys. Rev. B, in press (1991).

${ }^{42}$ W.W. Park, M.F. Becker, and R.M. Walser, Appl. Phys. Lett. 52, 1517 (1988).

${ }^{43}$ Y.J. Jeon, W.W. Park, M.F. Becker, and R.M. Walser, Mater. Res. Soc. Symp. Proc. 128, 551 (1989).

${ }^{44}$ E.P. Donovan, F. Spaepen, J.M. Poate, D.C. Jacobson, Appl. Phys. Lett. 55, 1516 (1989).

${ }^{45}$ S. Roorda, J.M. Poate, D.C. Jacobson, D.J. Eaglesham, B.S. Bennis, S. Dierker, W.C. Sinke, and F. Spaepen, Solid State Comm. 75, 197 (1990).

${ }^{46}$ S. Roorda and W.C. Sinke, Mater. Res. Soc. Symp. Proc. 205 (in press, 1991); and references within.

47W.C. Sinke, S. Roorda, and F. Saris, J. Mater. Res. $\underline{3}$, 1202 (1988).

${ }^{48}$ R.A. Street and D.K. Biegelsen, in The Physics of Hydrogenated Amorphous Silicon, edited by J.D. Joannopoulos and G. Lucovsky, (Springer-Verlag, Berlin, 1984), p. 195.

${ }^{49}$ S.T. Pantelides, Phys. Rev. Lett. 57, 2979 (1986).

${ }^{50}$ C.N. Waddell, W.G. Spitzer, J.E. Fredrickson, G.K. Hubler and T.A. Kennedy, J. Appl. Phys. 55, 4361 (1984).

${ }^{51}$ A.L. Greer, J. Non-Crystalline Solids 61/62, 737 (1984).

52B. Park, Ph.D. Thesis, Harvard University (1989).

${ }^{53}$ E. Nygren, B. Park, L.M. Goldman, and F. Spaepen, Appl. Phys. Lett. 56, 2094 (1990).

${ }^{54}$ H. Letaw, Jr., W.M. Portnoy, and L. Slifkin, Phys. Rev. 102, 636 (1956).

55M.W. Valenta and C. Ramasastry, Phys. Rev. 106, 73 (1957).

56H. Widmer and G.R. Gunther-Mohr, Helv. Phys. Acta 34, 635 (1961).

${ }^{57}$ G. Vogel, G. Hettich, and H. Mehrer, J. Phys. C $\underline{16}$, 6197 (1983).

58 M. Werner, Ph.D. Thesis, University of Stuttgart, Federal Republic of Germany (1984).

59 L. Kalinowski and R. Seguin, Appl. Phys. Lett. 35, 211 (1979); Appl. Phys. Lett. 36, 171 (1980).

60M. Werner, H. Mehrer, and H.D. Hochheimer, Phys. Rev. B32, 3930 (1985).

${ }^{61}$ M.J. Aziz, E. Nygren, W.H. Christie, C.W. White, and D. Turnbull, Mater. Res. Soc. Symp. Proc. 36, 101 (1985).

62F.P. Larkins and A.M. Stoneham, J. Phys. C 4, 143 (1971); ㄴ, 154 (1971).

${ }^{63}$ G.A. Baraff, E.O. Kane, and M. Schluter, Phys. Rev. B 21, 5662 (1980).

${ }^{64}$ U. Lindefelt, Phys. Rev. B 28, 4510 (1983).

65M. Scheffler, J.P. Vigneron, and G.B. Bachelet, Phys. Rev. B 31, 6541 (1985).

66A. Antonelli and J. Bernholc, Phys. Rev. B $\underline{40}$, 10643 (1989).

${ }^{67}$ G.D. Watkins, Deep Centers in Semiconductors, edited by S.T. Pantelides, (Gordon and Breach, New York, 1986), p. 147.

${ }^{68}$ W. Frank, U. Gosele, H. Mehrer, and A. Seeger, in Diffusion in Crystalline Solids, eds. G.E. Murch and A.S. Nowick (Academic, Orlando, Fl., 1984), p. 63.

${ }^{69}$ A. Witvrouw and F. Spaepen, Mater. Res. Soc. Symp. Proc. 205 (in press, 1991).

70V.J. Fratello, J.F. Hays, F. Spaepen, and D. Turnbull, J. Appl. Phys. 51, 6160 (1980). 
71F. Spaepen, Acta Metall. 26, 1167 (1978).

72T. Saito and I. Ohdomari, Philos. Mag. B 49, 471 (1984).

73D.T. Wu and M.J. Aziz (unpublished).

74I.R. Cox-Smith, H.C. Liang and R.O. Dillon, J. Vac. Sci. Technol. A 3, 674 (1985).

${ }^{75}$ L. Pauling, Nature of the Chemical Bond, Cornell U. Press, Ithaca NY (1960), p. 85.

76F. Spaepen, in Amorphous Materials: Modeling of Structure and Properties, ed. V. Vitek (TMS-AIME, New York, 1983, p. 265).

${ }^{77} \mathrm{D}$. Turnbull (unpublished).

78V. J. Fratello, J. F. Hays, and D. Turnbull, J. Appl. Phys. 51, 4718 (1980).

${ }^{79}$ Q. Williams and R.P. Jeanloz, Science 239, 902 (1988).

${ }^{80}$ R.J. Hemley, A.P. Jephcoat, H.K. Mao, L.C. Ming and M.H. Manghnani, Nature 334, 52 (1988).

81Y. Tsuchida and T. Yagi, Nature 347, 267 (1990).

${ }^{82}$ R.M. Walser and Y.-J. Jeon, Mat. Res. Soc. Symp. Proc. 205 (in press, 1991).

${ }^{83}$ R.M. Walser (private communication).

${ }^{84}$ L.E. Mosley and M.A. Paesler, J. Appl. Phys. 57, 2328 (1985).

${ }^{85}$ L.E. Mosley, P.J. Germaine, M.A. Paesler, and K. Zellama, J. Non-Cryst. Solids 59\&60, 273 (1983).

86Y.-J. Jeon, M.F. Becker, and R.M. Walser, "Ionization-Enhanced Solid Phase Epitaxy of Amorphous Silicon With Aluminum Impurities," Mat. Res. Soc. Symp. Proc. 205 (in press, 1991).

${ }^{87}$ R.A. Street, J. Non-Cryst. Sol. 77-78, 1 (1985); equation (3).

${ }^{88}$ S. Kalbitzer. G. Müller, P.G. Le Comber, and W.E. Spear, Phil. Mag, B41, 439 (1980).

${ }^{89}$ R.G. Elliman, S.T. Johnson, K.T. Short, and J.S. Williams, Mat. Res. Soc. Symp. Proc. 27, 229 (1984).

${ }^{90}$ For a review see F. Priolo and E. Rimini, Mat. Sci. Repts. $\underline{5}$, 319 (1990).

${ }^{91}$ K.A. Jackson, J. Mater. Res. $\underline{3}$, 1218 (1988).

${ }^{92}$ F. Priolo, C. Spinella, and E. Rimini, Phys. Rev. B 41, 5235 (1990).

${ }^{93}$ E. Nygren (unpublished).

${ }^{94}$ D.E. Polk, J. Non-Cryst. Solids 5, 365 (1971).

95P. Keating, Phys. Rev. 145, 637 (1966).

96J. Linnros and G. Holmen, J. Appl. Phys. 59, 1513 (1986).

${ }^{97}$ R.G. Elliman, J.S. Williams, W.L. Brown, A. Leiberich, D.M. Maher, and R.V. Knoell, Nucl. Instrum. Meth. B 19/20, 435 (1987).

${ }^{98}$ S.M. Sze, Physics of Semiconductor Devices, 2nd ed. (Wiley, New York, 1981), ch. 1. 
TABLE I. Candidate point defects governing crystal growth and conclusions regarding tenability of mechanisms.

\section{Defect Governing SPEG Authors}

\section{Conclusions}

Defects residing at c/a interface

Dangling bonds

Spaepen and Turnbulla

Plausible

Kink sites

Williams and Elliman $\mathrm{b}$

special case of dangling bond

mechanism

Defects residing in crystal

Vacancies

Csepregi et al. ${ }^{\mathrm{c}}$, Suni et al. ${ }^{\mathrm{d}}$

Highly Implausible (Si);

Impossible (Ge)

Interstitials

Highly Implausible

Defects residing in amorphous

Dangling bonds

Floating bonds

any other point defect in
Mosley et al. ${ }^{\mathrm{e}}$

Pantelides $\mathrm{f}$

Narayang, Licoppe and Nissim ${ }^{\mathrm{h}}$
Impossible

Impossible amorphous phase

asee ref. 12 .

bSee ref. 13.

cSee ref. 2.

dSee ref. 6.
eSee ref. 15.

fSee ref. 16. gSee ref. 14.

hSee ref. 17. 
TABLE II. Measured growth rates for Ge.

\begin{tabular}{lll}
\hline \hline Temperature $\left({ }^{\circ} \mathrm{C}\right)$ & Pressure $(\mathrm{GPa})$ & $\mathrm{V}(\mathrm{nm} / \mathrm{sec})$ \\
\hline 300 & 2.7 & 0.032 \\
& 3.65 & 0.111 \\
& 4.6 & 0.472 \\
& 4.75 & 0.375 \\
& & \\
325 & 0.0 & $0.0068 \mathrm{a}$ \\
& 0.90 & 0.029 \\
1.26 & 0.050 \\
2.20 & 0.253 \\
& 2.80 & 0.30 \\
& 2.87 & 0.50 \\
& 3.00 & 0.360 \\
& 3.50 & 0.64 \\
& 4.32 & 1.90 \\
& 5.02 & 2.80 \\
& & \\
& 0.0 & 0.02 \\
345 & 0.60 & 0.055 \\
& 1.40 & 0.162 \\
& 2.30 & 0.580 \\
& 4.20 & 6.10 \\
& 0.0 & 0.086 \\
365 & 0.63 & 0.195 \\
& 1.26 & 0.338 \\
1.50 & 0.46 \\
2.60 & 1.90 \\
\hline \hline
\end{tabular}

aEstimated value; see ref. 28.

TABLE III. Measured growth rates for undoped Si.

\begin{tabular}{lll}
\hline \hline Temperature $\left({ }^{\circ} \mathrm{C}\right)$ & Pressure $(\mathrm{GPa})$ & $\overline{\mathrm{v}(\mathrm{nm} / \mathrm{sec})}$ \\
\hline 566 & 0.0 & 0.1 \\
& & \\
550 & 0.0 & 0.035 \\
& 0.0 & 0.037 \\
& 2.7 & 0.1284 \\
& & \\
530 & 0.0 & 0.020 \\
& 0.50 & 0.026 \\
1.10 & 0.033 \\
& 1.40 & 0.040 \\
& 1.70 & 0.048 \\
& 3.20 & 0.105 \\
520 & 0.0 & 0.0120 \\
& 1.10 & 0.027 \\
& 5.00 & 0.126
\end{tabular}


TABLE IV. High pressure anneals for doped and compensated Si.

\begin{tabular}{|c|c|c|}
\hline$\overline{\text { Dopant }}$ & Temperature $\left({ }^{\circ} \mathrm{C}\right)$ & $=\overline{\text { Pressure }(\mathrm{GPa})}$ \\
\hline \multirow[t]{2}{*}{$75 \mathrm{As}^{+}, 75 \mathrm{keV}, 1 \times 10^{15} / \mathrm{cm}^{2}$} & 530 & 0.0 \\
\hline & & $\begin{array}{l}1.7 \\
3.0\end{array}$ \\
\hline \multirow{2}{*}{${ }^{11} \mathrm{~B}^{+}, 35 \mathrm{keV}, 1.5 \times 10^{15} / \mathrm{cm}^{2}$} & 520 & 0.0 \\
\hline & & $\begin{array}{l}2.0 \\
2.8\end{array}$ \\
\hline \multirow[t]{2}{*}{${ }^{31} \mathrm{P}^{+}, 110 \mathrm{keV}, 1.5 \times 10^{15} / \mathrm{cm}^{2}$} & 520 & 0.0 \\
\hline & & 2.6 \\
\hline \multirow[t]{2}{*}{${ }^{11} \mathrm{~B}^{+}$and ${ }^{31} \mathrm{P}^{+}$, as above } & 520 & 0.0 \\
\hline & & 2.5 \\
\hline
\end{tabular}

TABLE V. Distribution of nearest neighbor changes during migration of vacancy and of dangling bond.

\begin{tabular}{|c|c|c|c|c|}
\hline $\begin{array}{l}\text { number of } \\
\text { atoms that: }\end{array}$ & $\begin{array}{l}\text { lose } 1 \\
\text { neighbor }\end{array}$ & gain 1 neighbor & $\begin{array}{l}\overline{\text { lose } 1 \text { neighbor and }} \\
\text { gain } 1 \text { neighbor }\end{array}$ & $\begin{array}{l}=\overline{\text { lose } 3 \text { neighbors and }} \\
\text { gain } 3 \text { neighbors }\end{array}$ \\
\hline vacancy migration & 3 & 3 & 0 & 1 \\
\hline $\begin{array}{l}\text { dangling bond } \\
\text { migration }\end{array}$ & 1 & 1 & 1 & 0 \\
\hline
\end{tabular}

TABLE VI. Lower limits on number of atoms crystallized per defect impingement .on interface, from equation (12).

\begin{tabular}{lll}
\hline \hline defect type & $\overline{\mathrm{Si}, 803 \mathrm{~K}}$ & $\overline{\mathrm{Ge}, 600 \mathrm{~K}}$ \\
\hline defect in bulk crystal & $\mathrm{N}_{\mathrm{r}}>3 \times 10^{8}$ & $\mathrm{~N}_{\mathrm{r}}>10^{8}$ \\
$\begin{array}{l}\text { vacancy- and interstitial-like defects } \\
\text { in bulk amorphous phase }\end{array}$ & $\mathrm{N}_{\mathrm{r}}>7$ & $\mathrm{~N}_{\mathrm{r}}>3 \times 10^{4}$ \\
$\begin{array}{l}\text { dangling and floating bonds in bulk } \\
\text { amorphous phase }\end{array}$ & $\mathrm{N}_{\mathrm{r}}>2$ & $\mathrm{~N}_{\mathrm{r}}>1 \times 10^{4}$ \\
\hline \hline
\end{tabular}


Figures

(a)

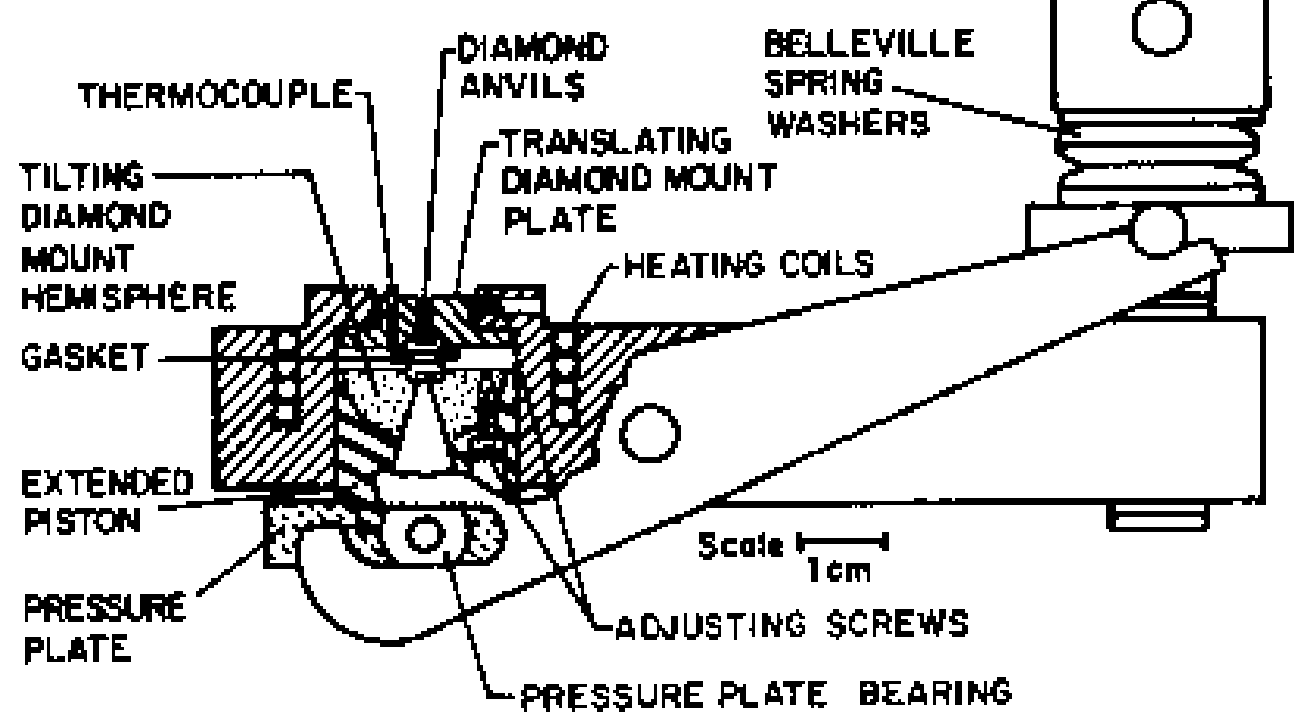

(b)

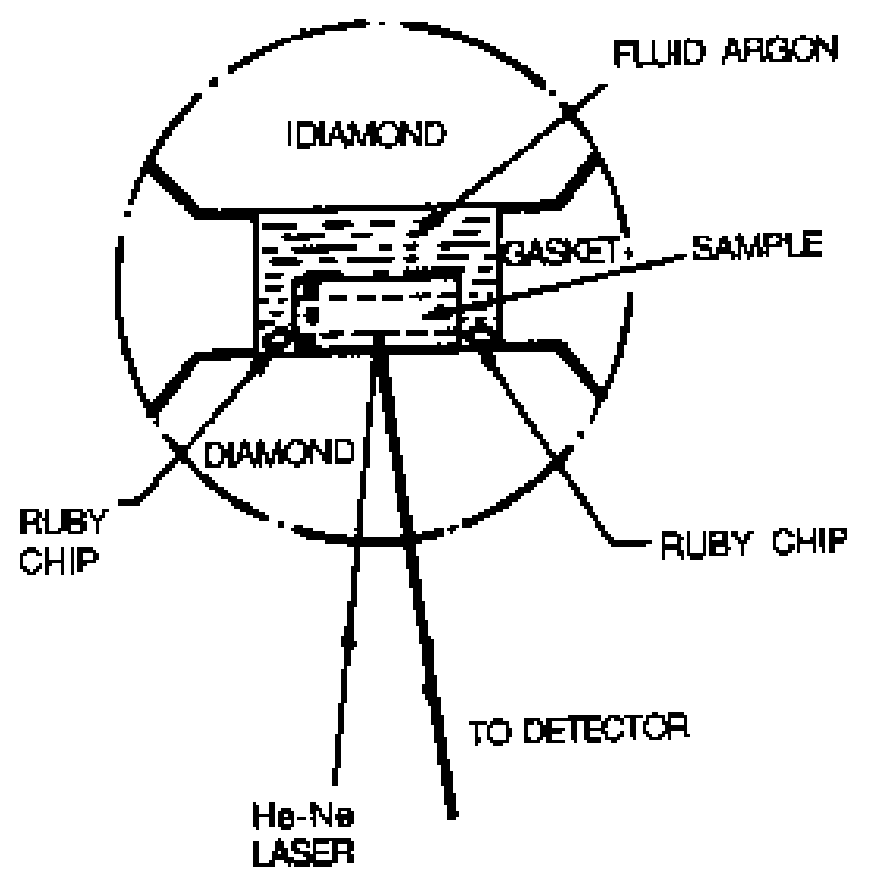

FIG. 1. (a) Schematic of the diamond anvil cell used in this study; (b) expanded sketch of the sample chamber. 


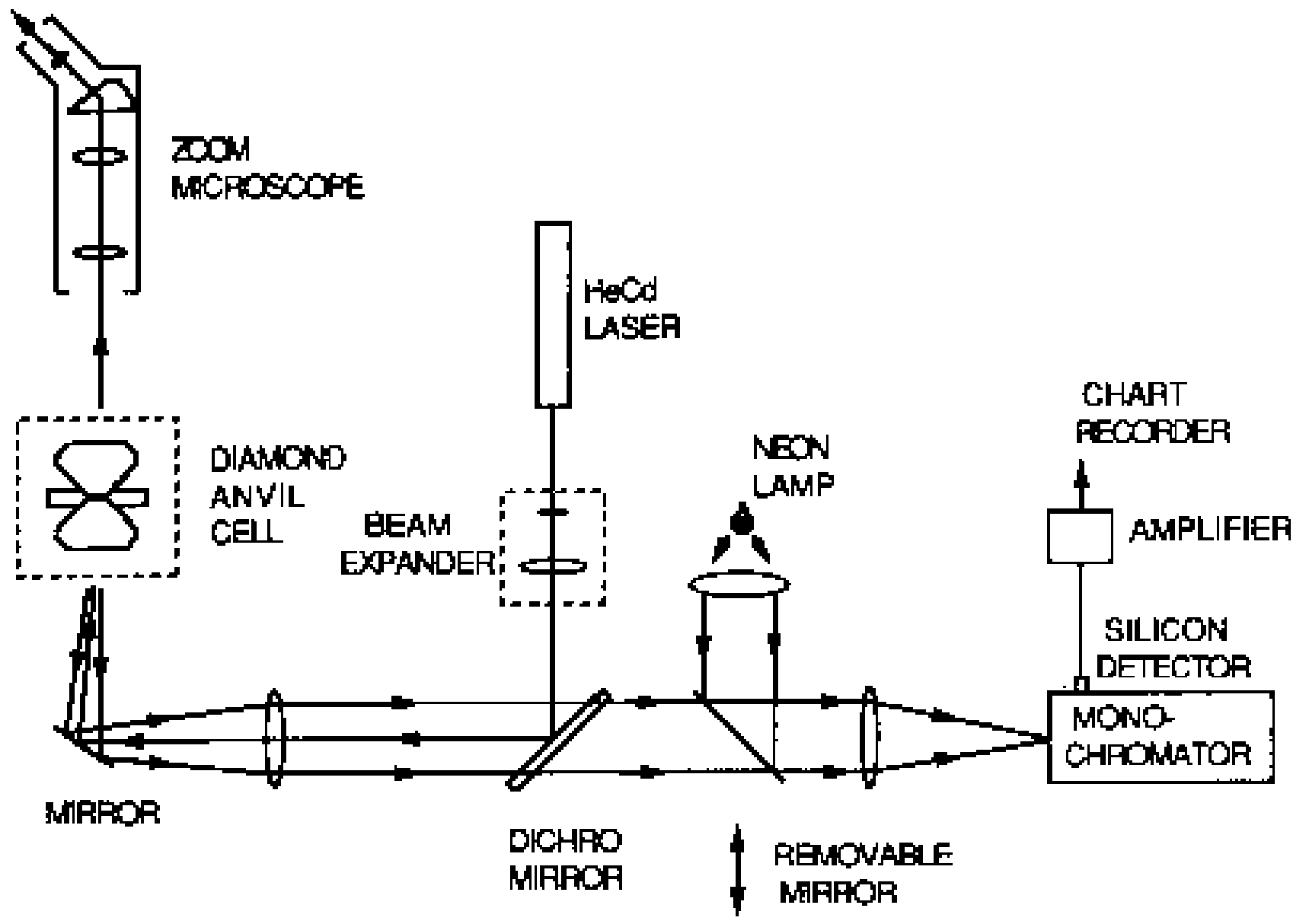

FIG. 2. Optical system used for pressure measurement with ruby fluorescence. 


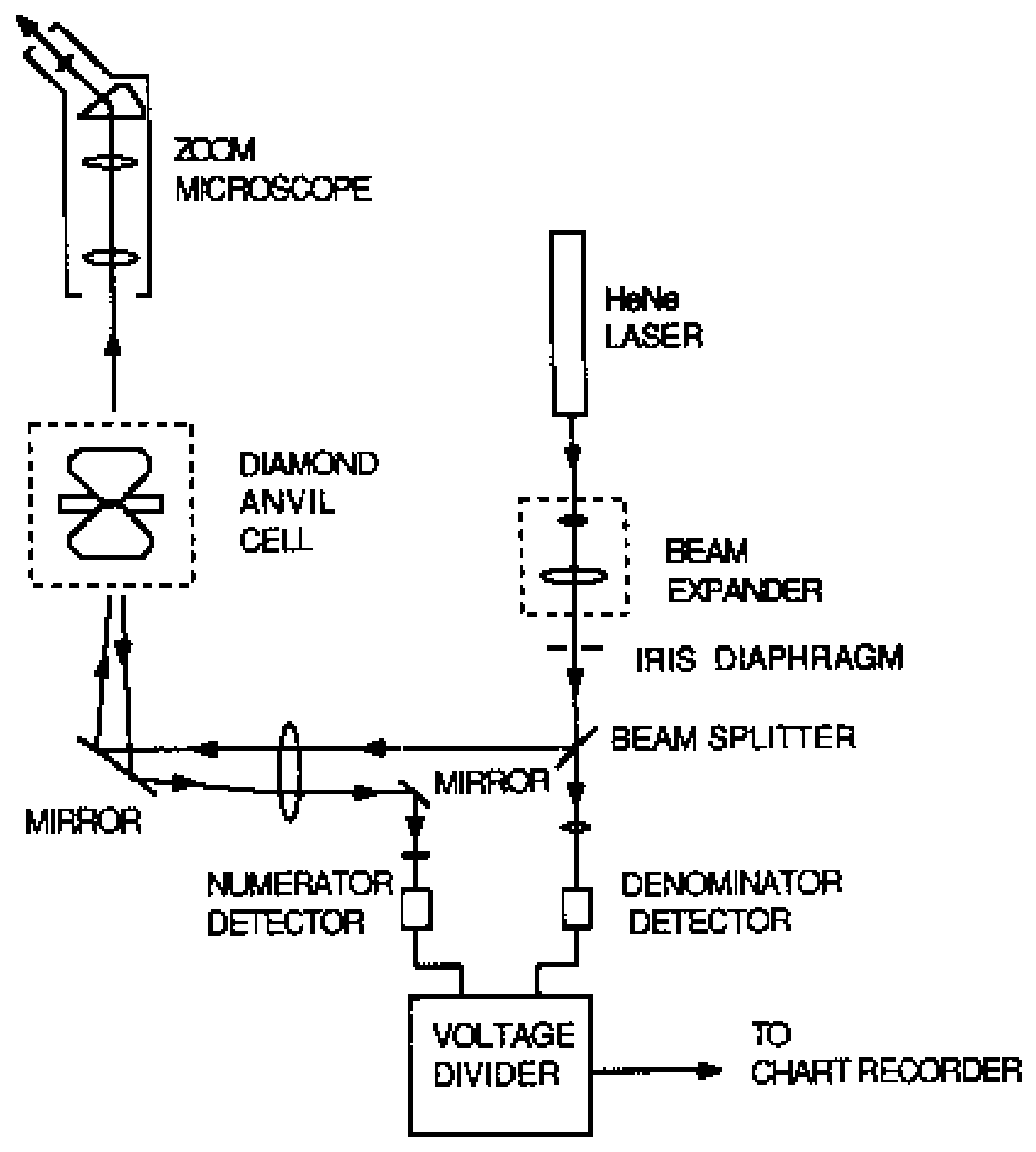

FIG. 3. Optical system for time-resolved reflectivity measurements of $\mathrm{Si}$ and Ge SPEG rate. 

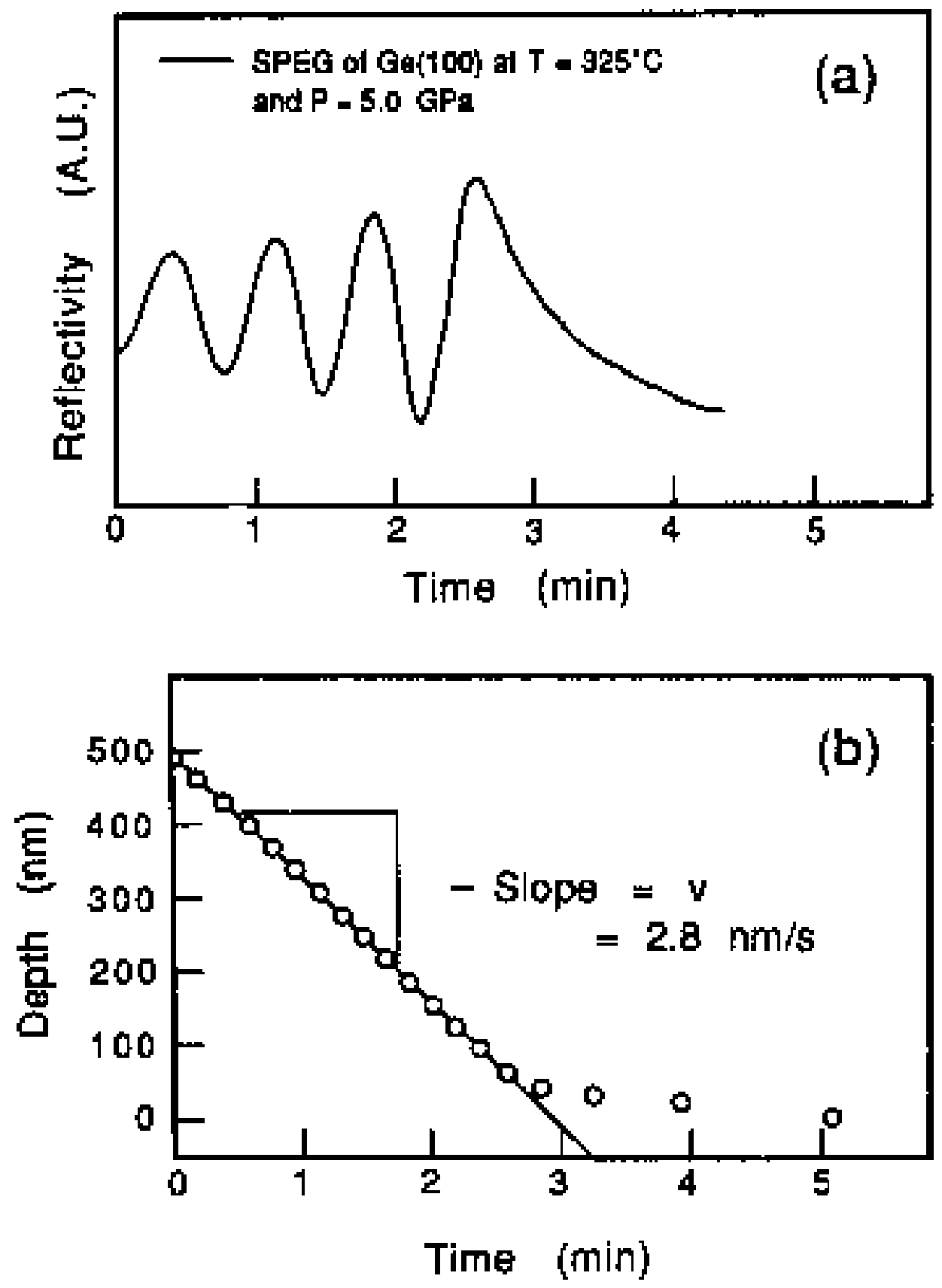

FIG. 4. (a) Time-resolved reflectivity from Ge SPEG; (b) Amorphous layer thickness vs time determined from maxima, minima, and midpoints in TRR trace. 


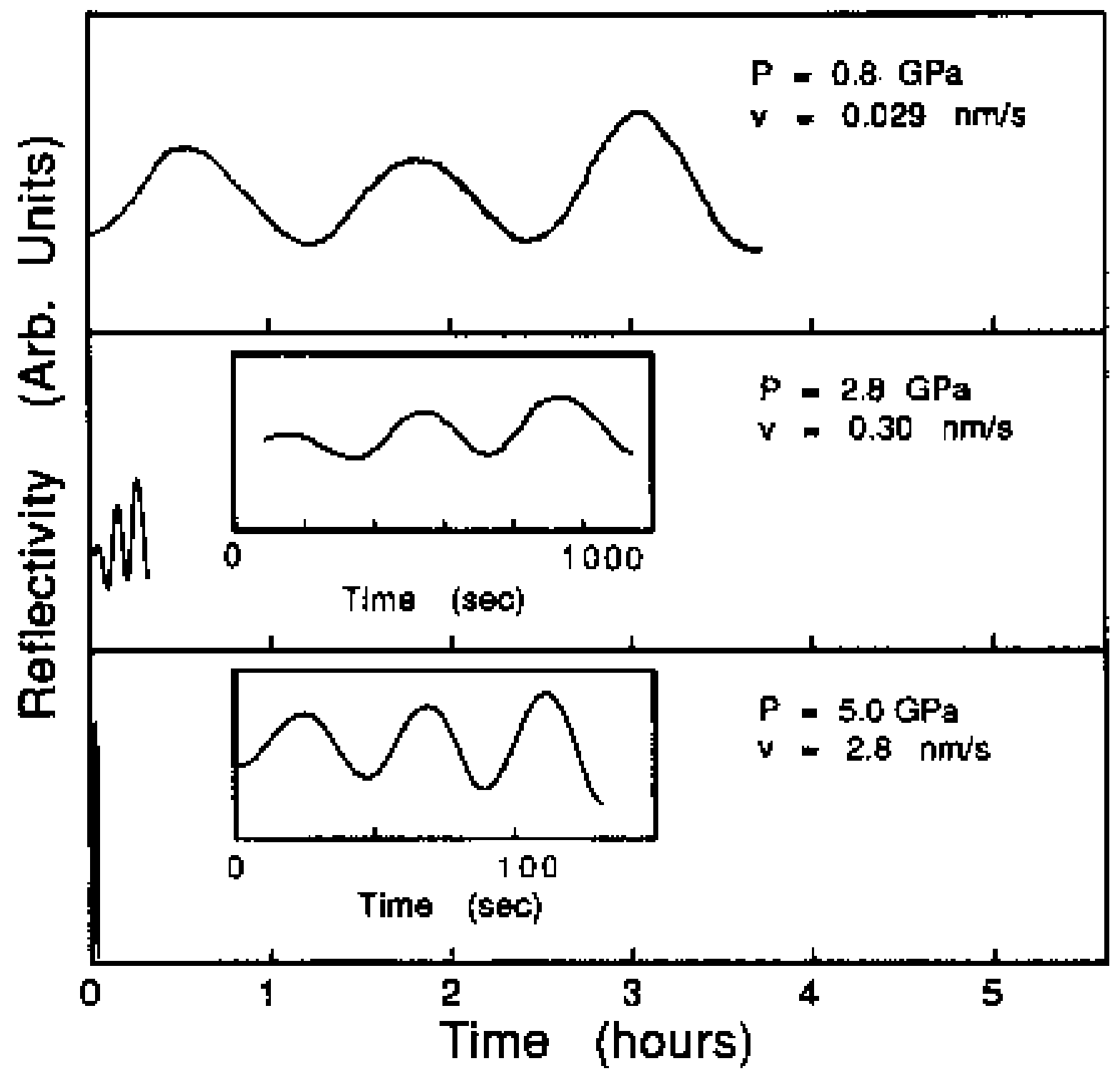

FIG. 5. TRR from SPEG of $\mathrm{Ge}(100)$ at $325^{\circ} \mathrm{C}$ under various pressures. 


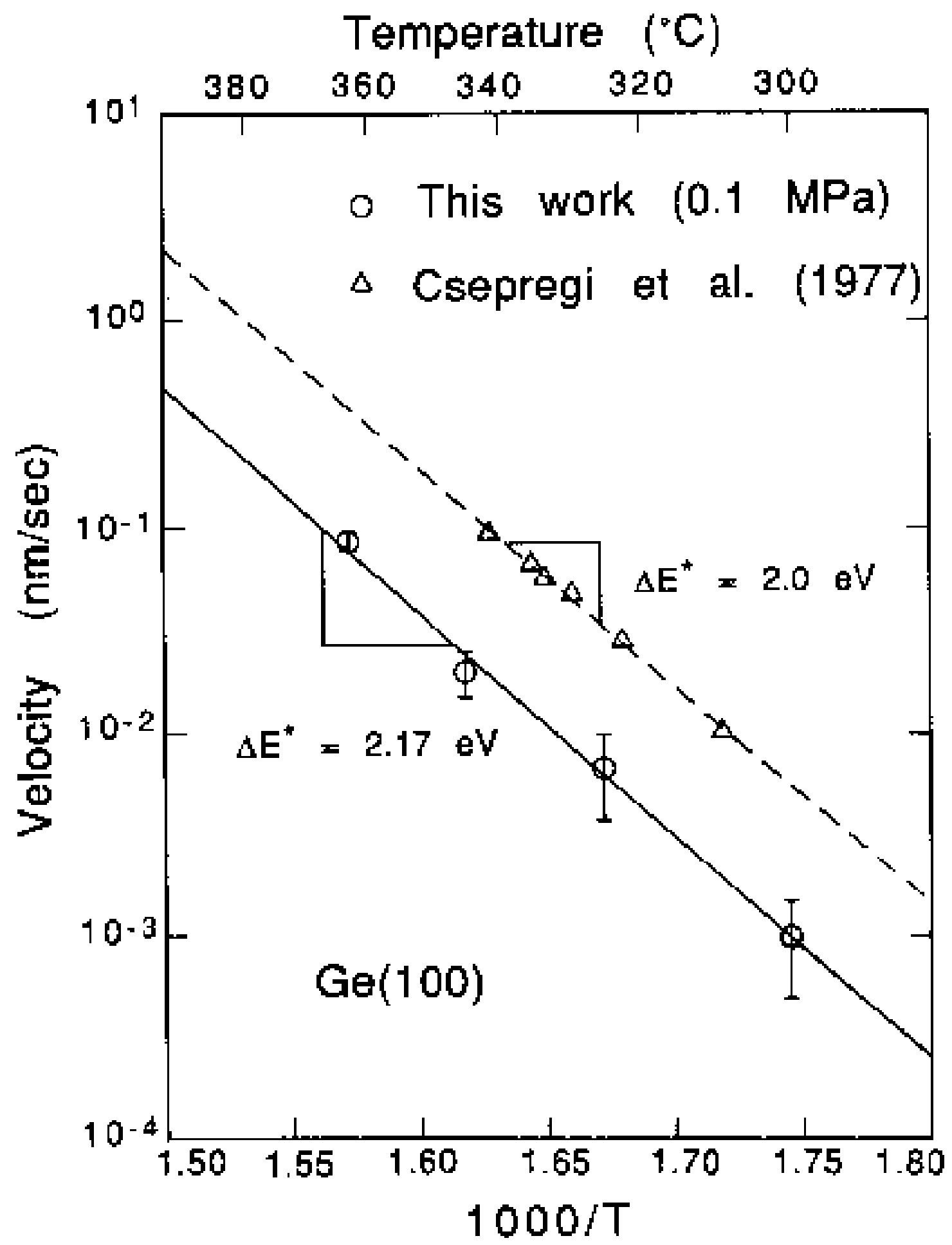

FIG. 6. Temperature dependence of Ge SPEG rate from this study and from that of Csepregi et al. (see Ref. 2). 


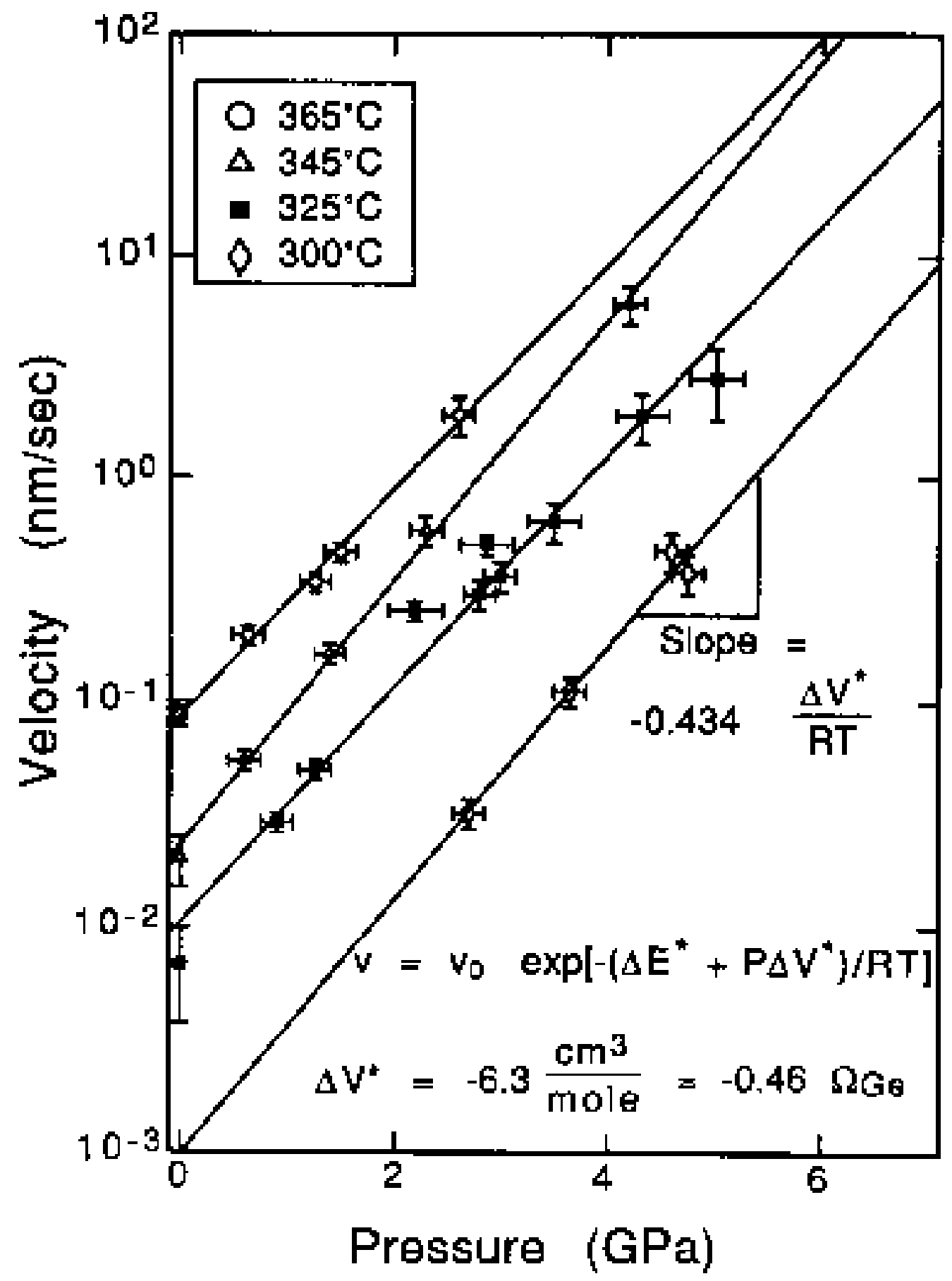

FIG. 7. Ge growth rate vs pressure. The average activation volume is $-0.46 \Omega_{\mathrm{CTC}}$ 

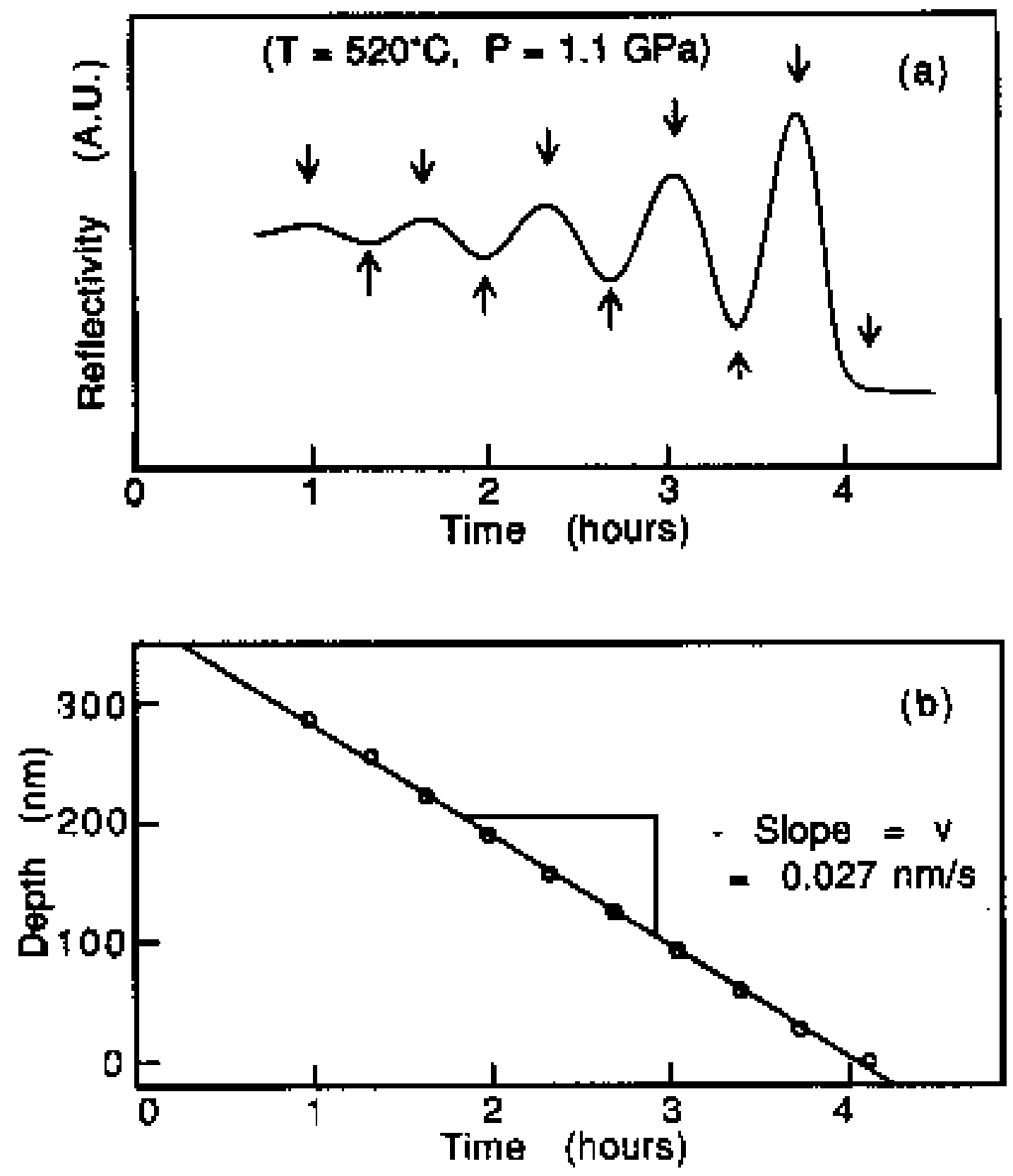

FIG. 8. (a) TRR from SPEG of $\mathrm{Si}(100)$ at high pressure; (b) Amorphous thickness vs time determined from maxima and minima in TRR trace. 


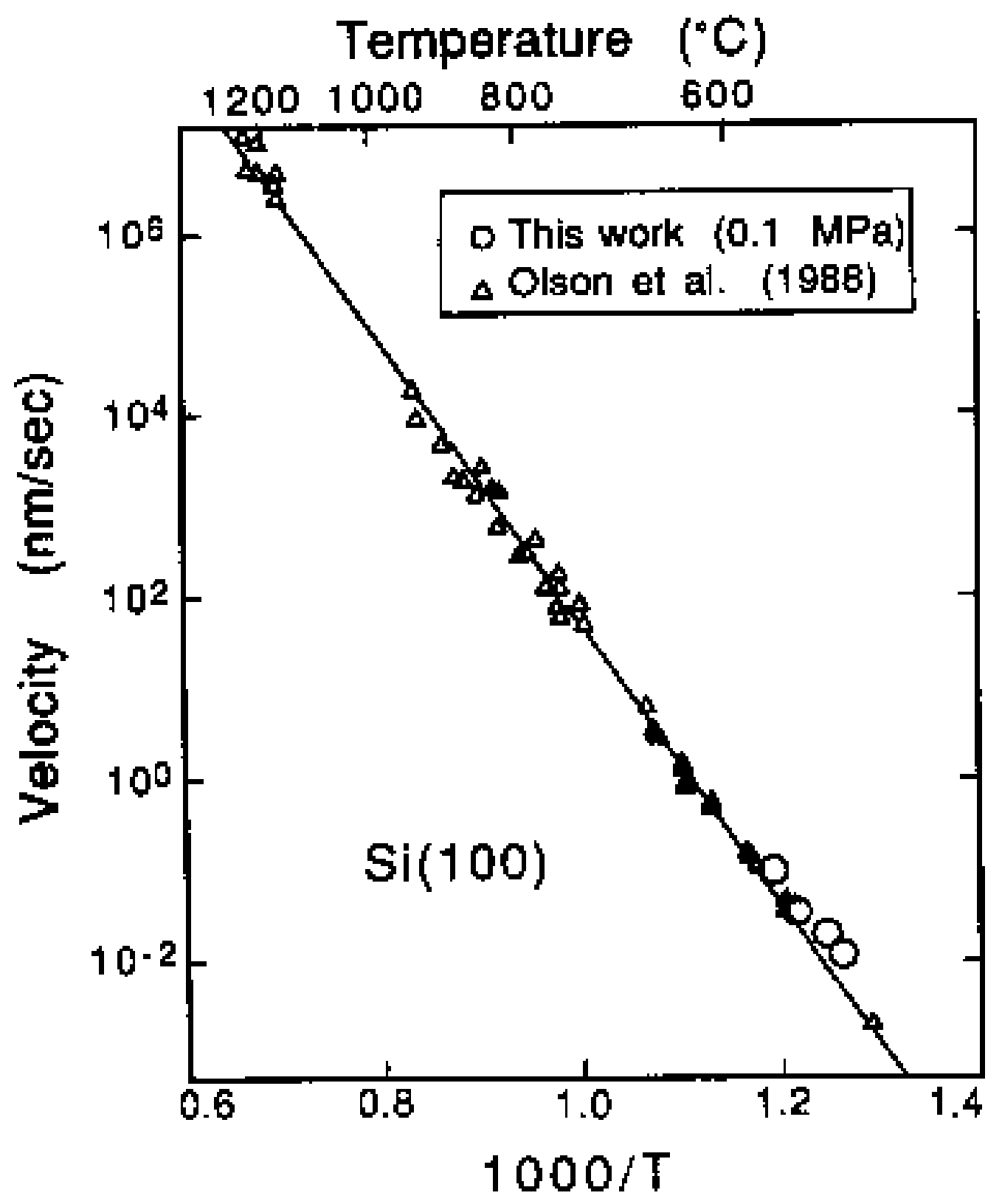

FIG. 9. Temperature dependence of Si SPEG rate at ambient pressure from this work and from Olson et al. (see Ref. 3). 


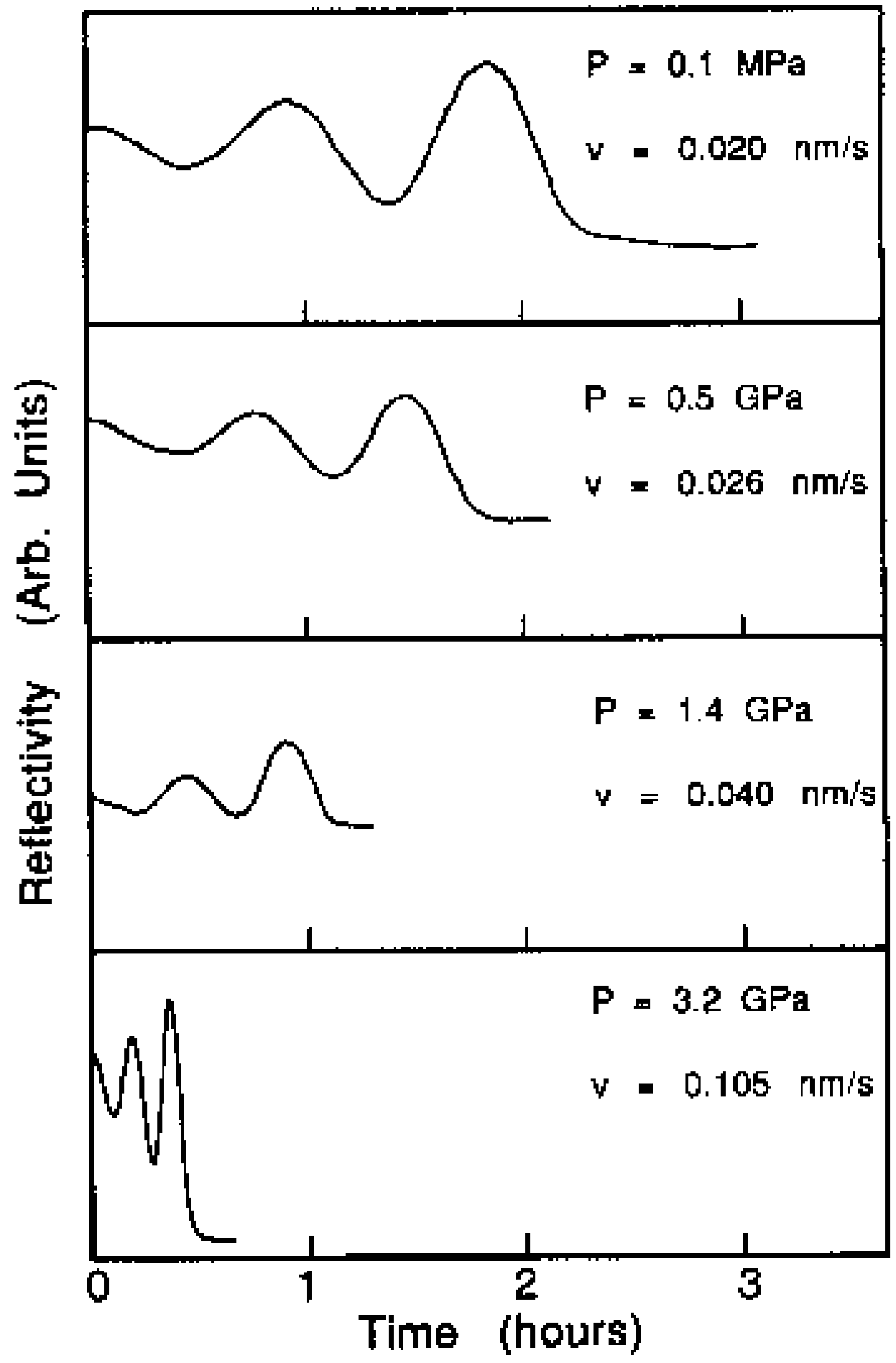

FIG. 10. TRR from SPEG of Si(100) at $530^{\circ} \mathrm{C}$ under various pressures. 


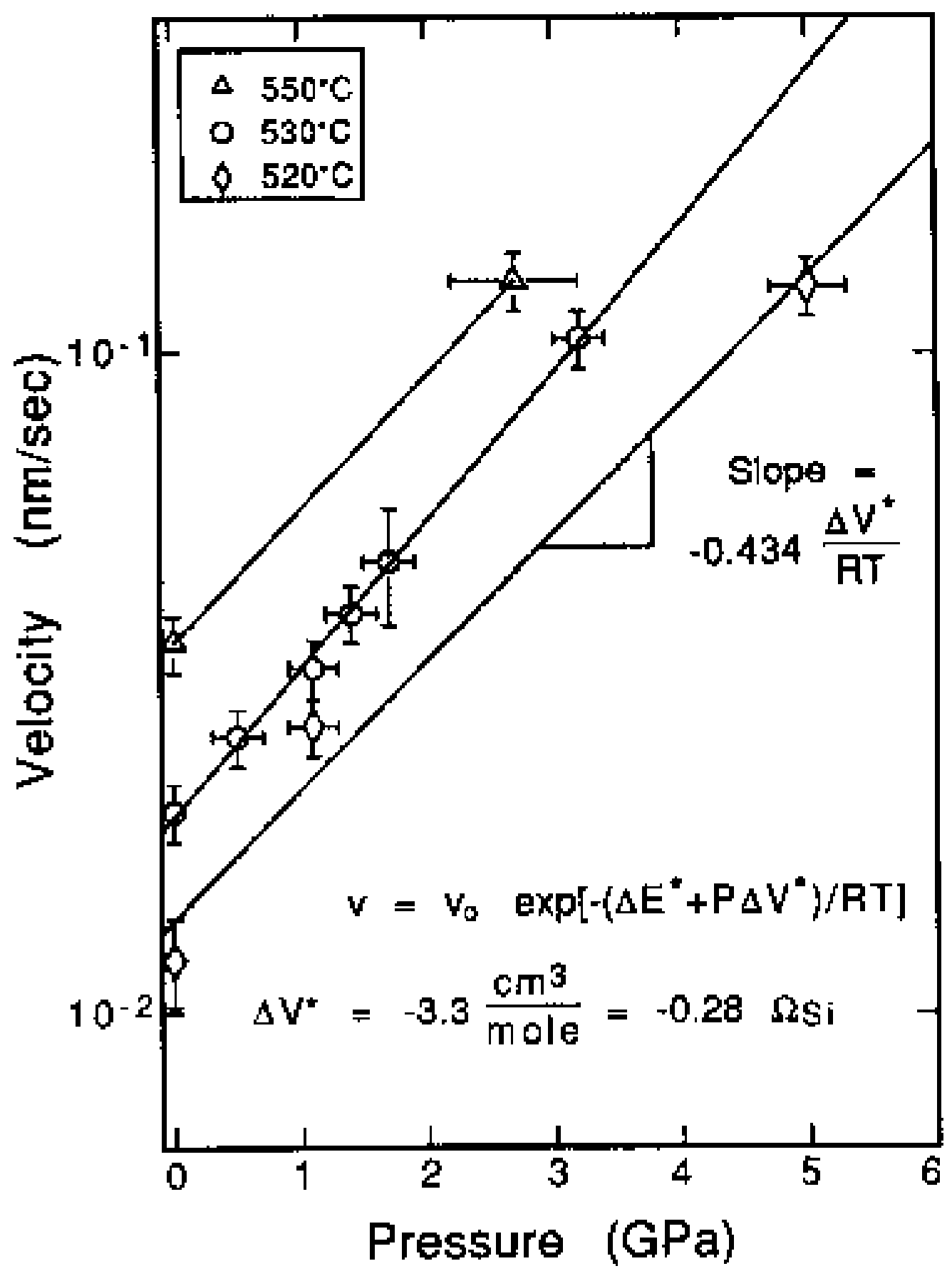

FIG. 11. Si growth rate vs pressure. The average activation volume is $-0.28 \Omega_{\mathrm{si}}$. 


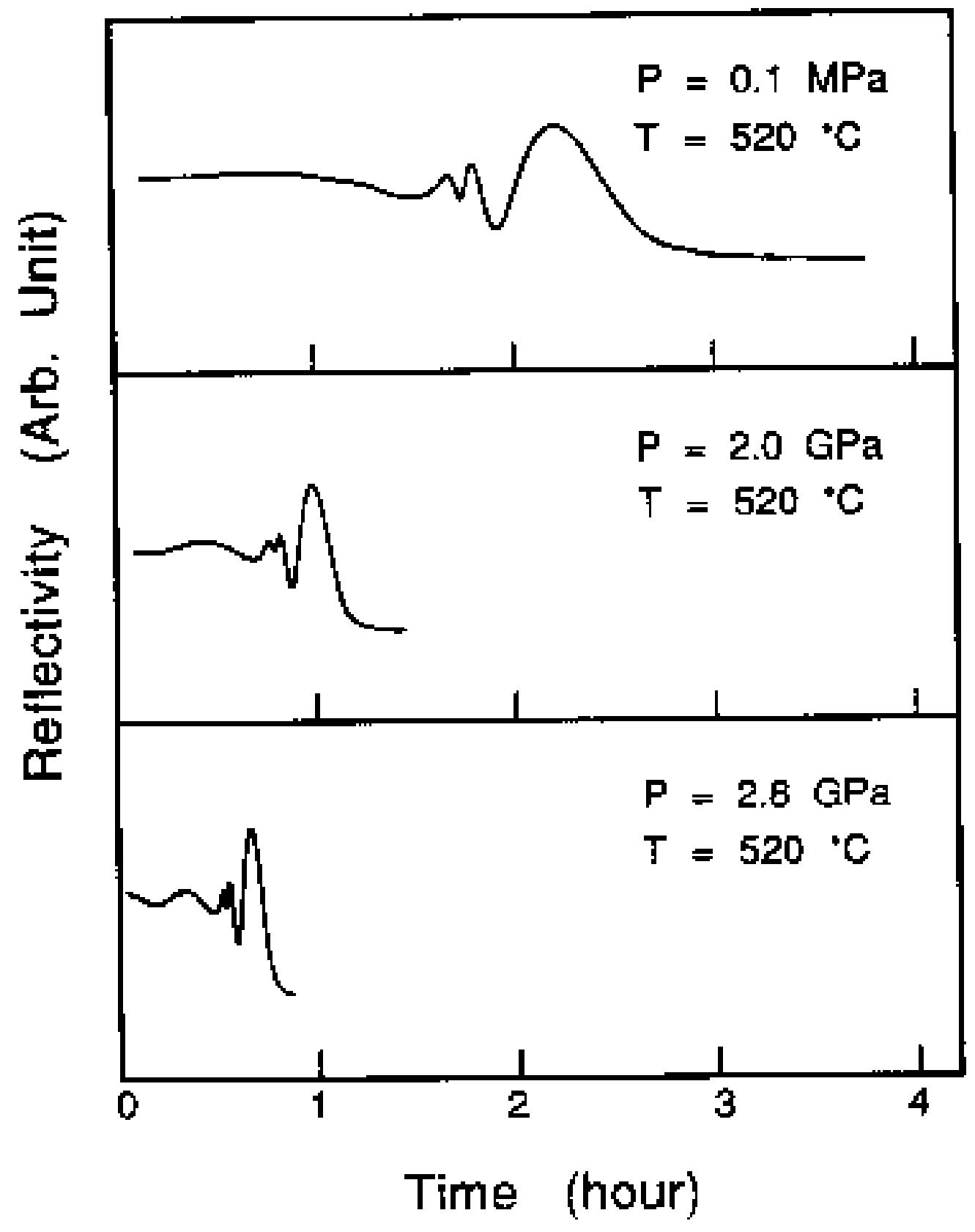

FIG. 12. TRR traces from B-doped silicon grown at various pressures. 


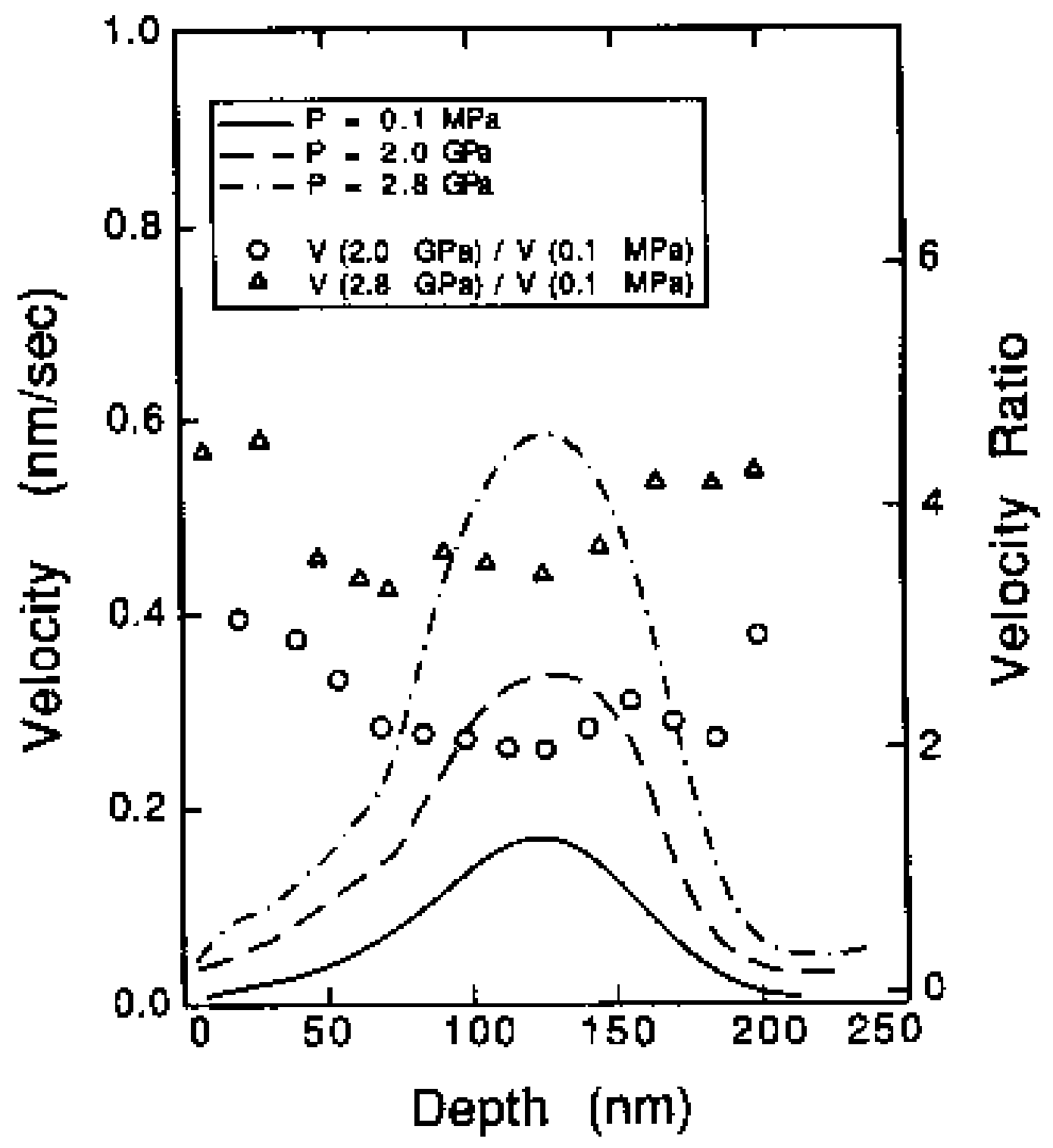

FIG. 13. Growth rate (curves, referred to left-hand scale) vs depth for B-doped silicon; ratios (points, referred to right-hand scale) of rate at high pressure to rate at ambient pressure at the same depth. 


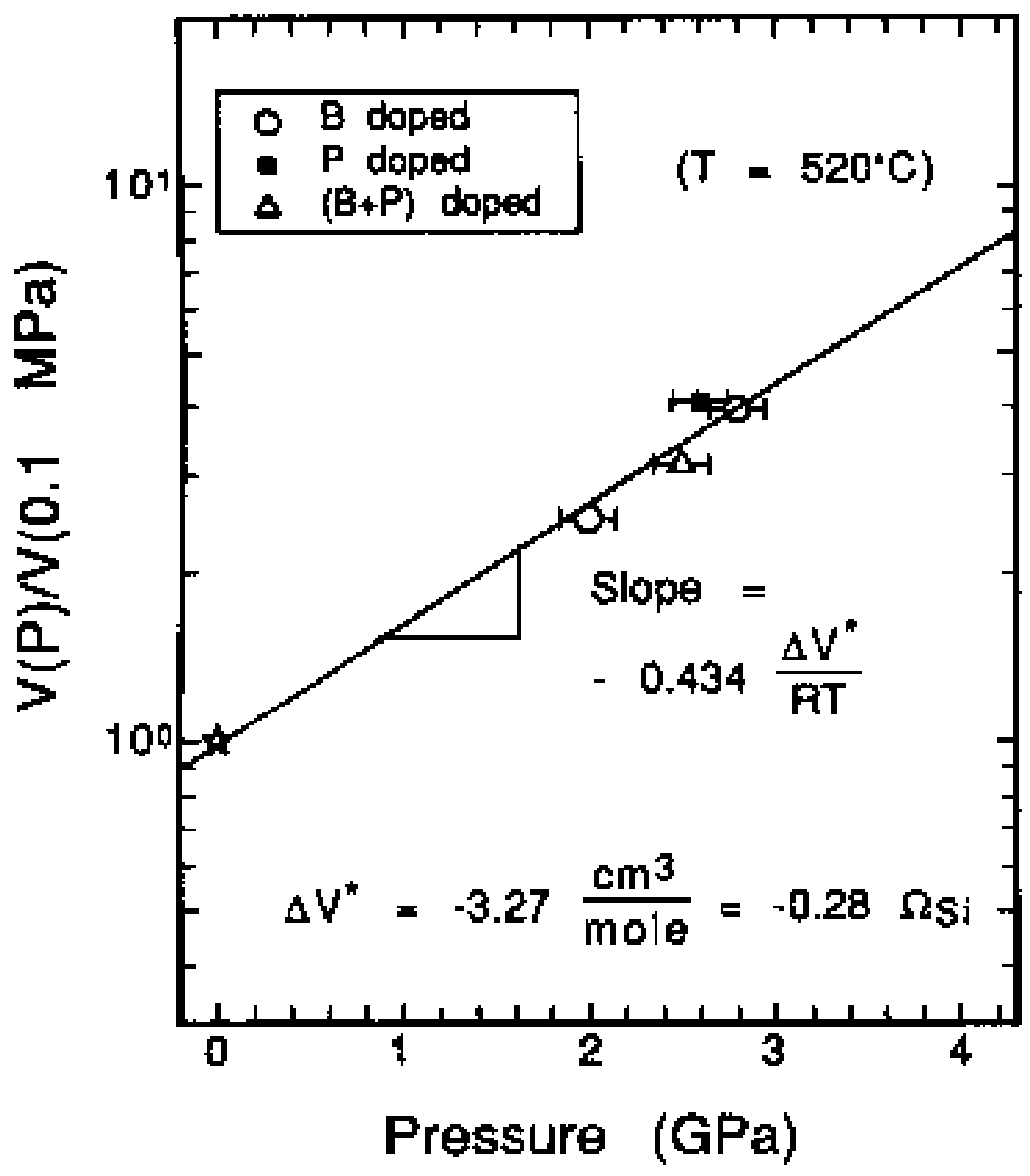

FIG. 14. Pressure dependence of growth rates, nomalized by anbientpressure values, for doped and compensated $\mathrm{Si}$. 


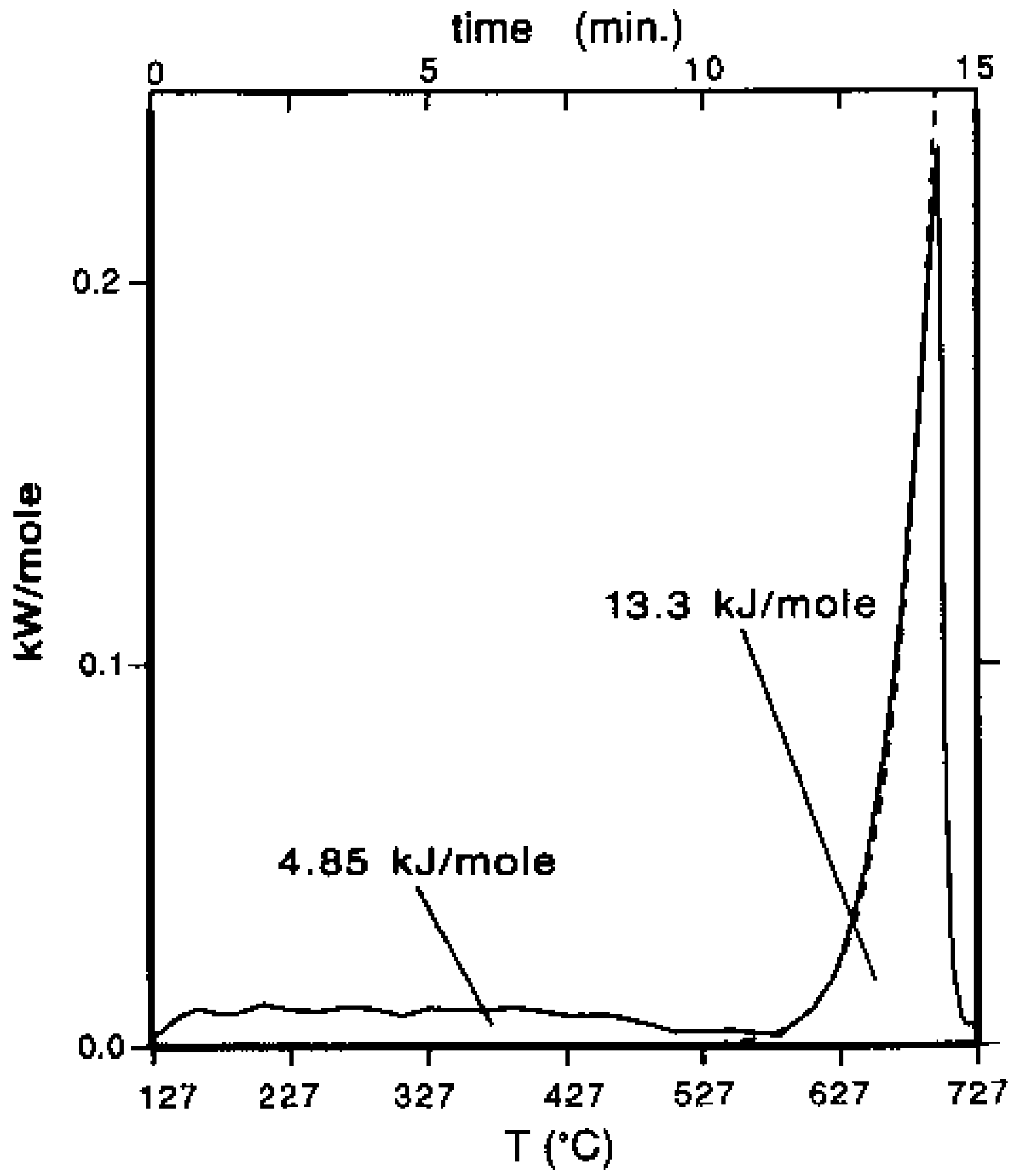

FIG. 15. Enthalpy release from $a-S i$, from Donovan et al. (see Ref. 44). Enthalpy release below $\sim 550^{\circ} \mathrm{C}$ is due to structural relaxation of $a-\mathrm{Si}$. The large peak is the heat of crystallization. 


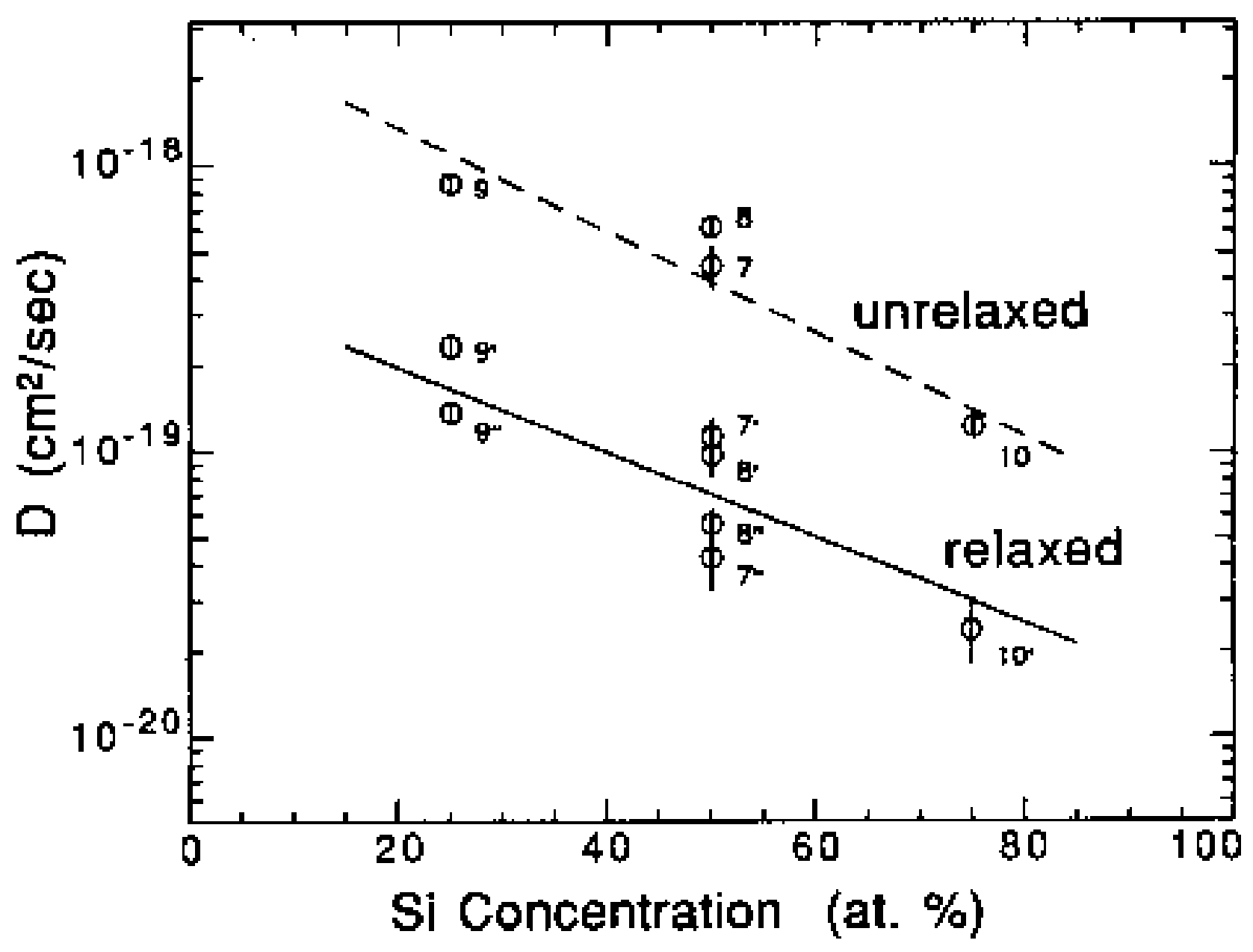

FIG. 16. Effect of amorphous-phase relaxation on interdiffusivity of $a-\mathrm{Si} /$ $a$-Ge multilayers at $427^{\circ} \mathrm{C}$, from Park (see Ref. 52). Four different samples are distinguished by different numbers. 


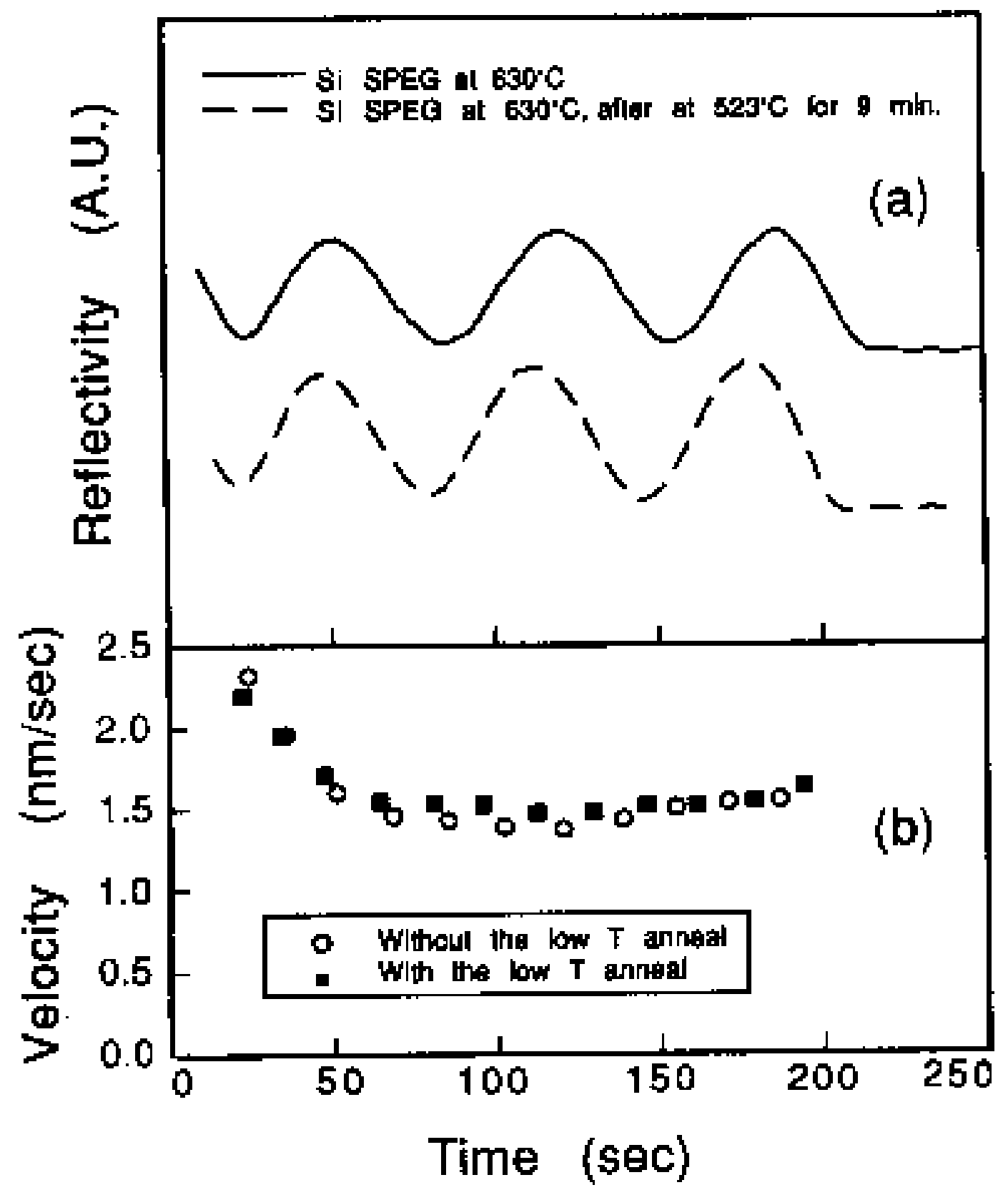

FJG. 17. Absence of effect of amorphous-phase relaxation on ambientpressure Si SPEG rate. (a) TRR traces from samples with and without low-temperature preanneal for structural relaxation; (b) indistinguishable velocity vs time curves for both samples. 


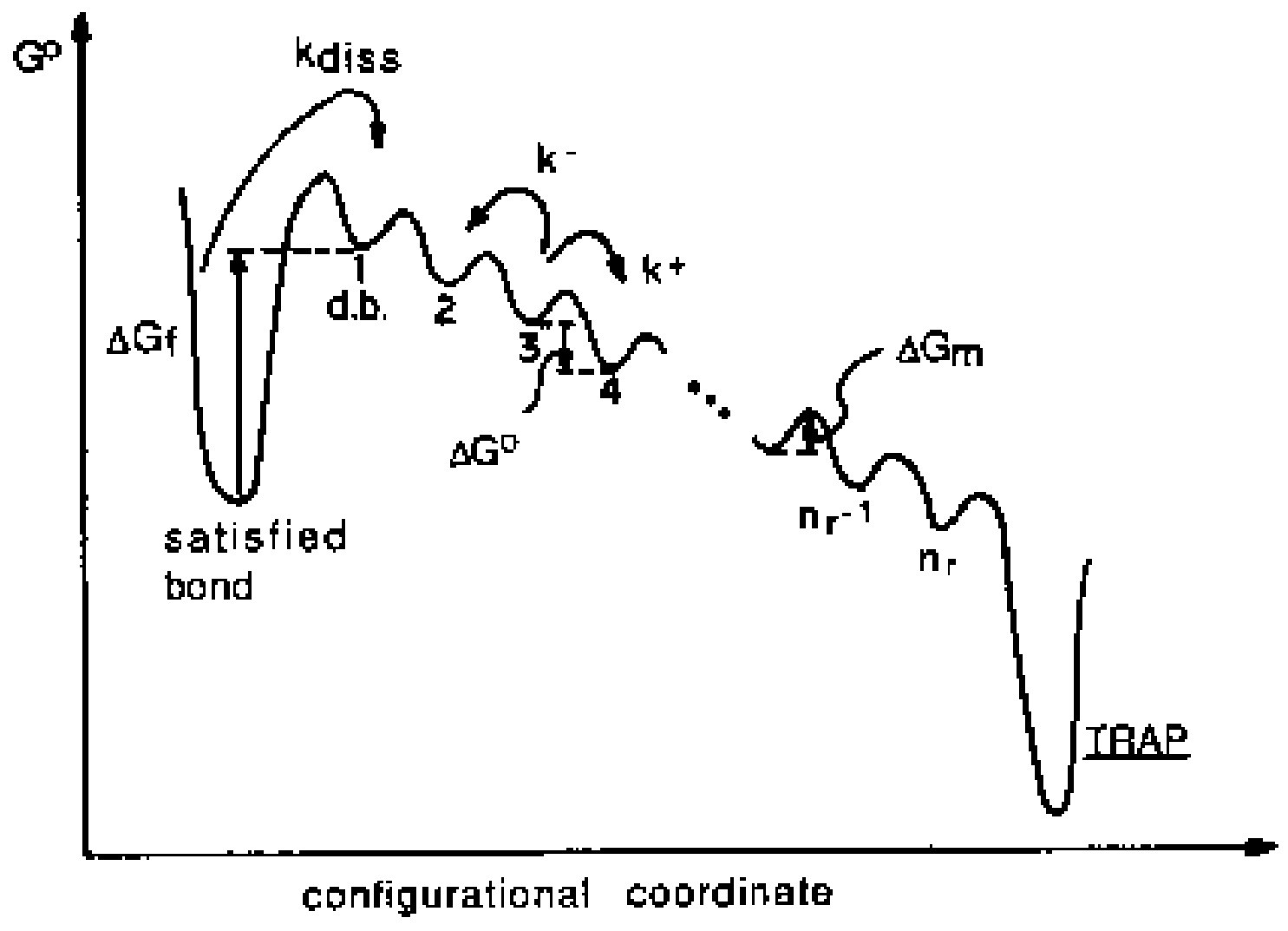

FIG. 18. Standard free energy vs configurational coordinate for breaking, moving, and trapping dangling bonds. 


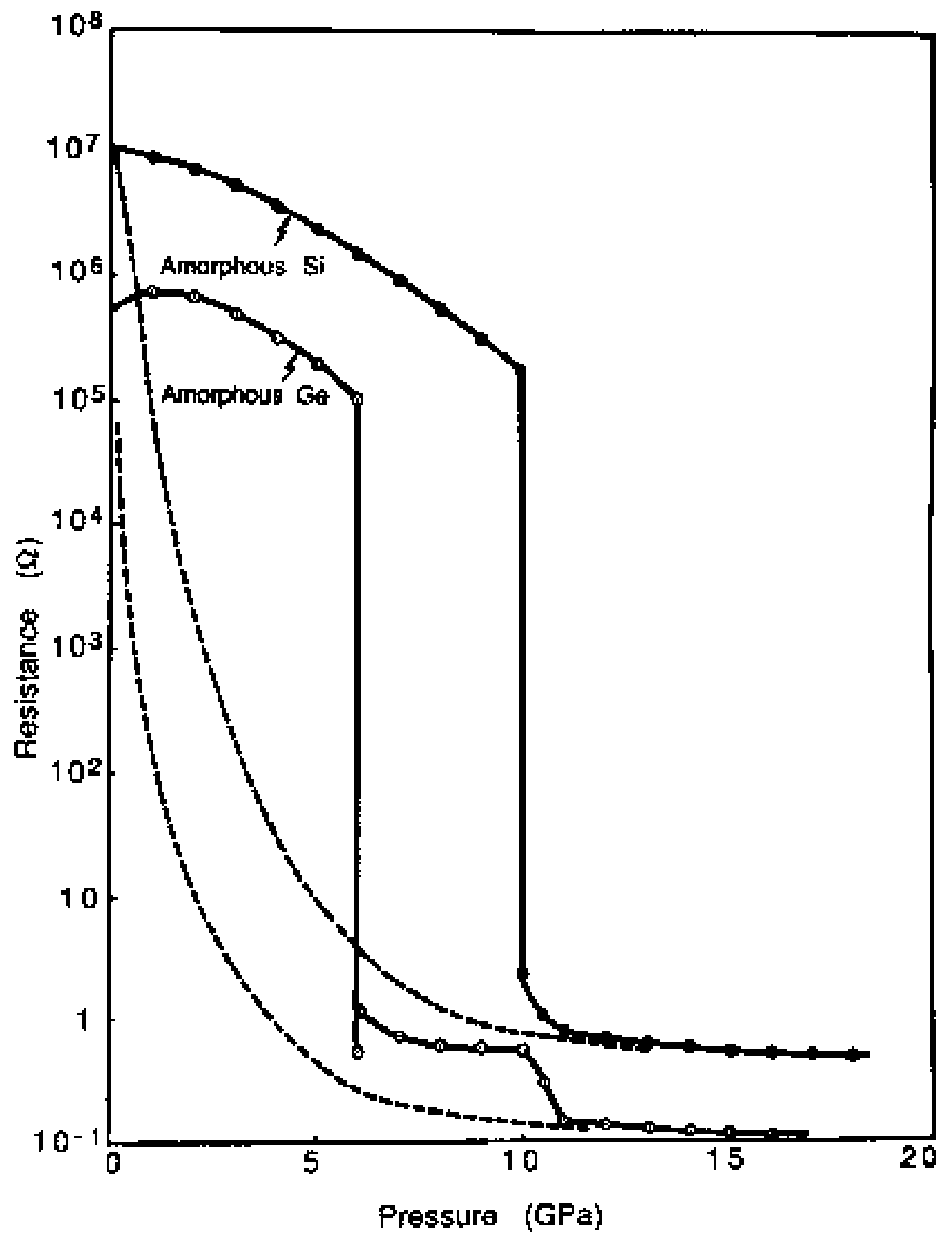

FIG. 19. Manifestation of the predicted free-energy catastrophe? Metallization of $\mathrm{Si}$ and $\mathrm{Ge}$ at high pressure, from Shimomura et al. (see Ref. 39). 